\title{
Rheological damping of slender rods
}

\author{
Pau Trubat*1, Climent Molins ${ }^{1}$ \\ pau.trubat.casal@upc.edu, climent.molins@upc.edu
}

1. Department of Civil and Environmental Engneering, Universitat Politècnica de Catalunya (UPC BarcelonaTech), C/Jordi Girona 1-3, 08034, Barcelona, Spain

* Corresponding author

\section{Keywords:}

Finite element model, slender rod, mooring system, cable, material damping, axial damping, bending damping, rheological damping, offshore

\begin{abstract}
Efficient and accurate modelling of mooring and cable systems for Floating Offshore Wind Turbines are a key factor in the coupled dynamic analysis for the realistic assessment of such floating structures. In order to improve the modelling of the mooring and cable systems, a new extension of the slender rod finite element model proposed by Garret with the inclusion of rheological damping is presented. The model takes into account the damping produced for the rod material in both the axial and the bending forces. Derivation of the axial and bending damping forces is presented and assessed in terms of the critical damping. The implementation of the model is presented and tested through three verification simulations and two validation examples, which show a good agreement when comparing with experimental results and prove the capacity and robustness of the model.
\end{abstract}

\section{Introduction}

Renewable energy market is expected to reach the $30 \%$ of the power production worldwide by 2022 , and wind and solar will represent the $80 \%$ of the renewable capacity growth [1]. The main wind energy resources are located offshore with around $80 \%$ placed in deep waters, which present larger and more consistent winds, but where bottom fixed platforms are not economically viable due to the depths are larger than $60 \mathrm{~m}$, [2]. Thus, Floating Offshore Wind Turbine (FOWT) is the proper technology to take advantage of this resource, but its technology readiness level is not already fully mature.

One of the key factors for a proper implementation of FOWT is a cost reduction in the mooring line solutions, electrical cables and grid connections, [2]. This cost reduction must outcome from new optimized mooring systems and as well from new advanced modelling tools which allow to predict more accurately all the stages of the life cycle of the FOWT, and particularly the reliability of the mooring and cable systems [3], [4].

The first dynamic model for mooring lines and cable was the lumped mass method [5]. Lumped Mass Method divides the mooring line in a number of elements, where its mass, internal forces and external forces are lumped in the nodes of the elements. Later, material elasticity was included by a spring system that connects consecutive lumped masses and the floor contact was also added [6]. On the other hand, line characteristics such as internal damping, bending and torsional stiffness were neglected. The 
equations of motions were derived by applying dynamic equilibrium and stress-strain deformations on each node, which were solved in the time domain using finite difference schemes.

A second approach to the spatial discretization of the mooring line is based on the finite differences. The main difference between the finite differences and the lumped mass method is the initial assumption of the element description for deducing the equations. Finite differences are based on a small differential element while lumped mass method uses a finite discrete element. Albow [7] proposed a method that discretizes the problem in both space and time using finite differences. This method uses a second-order implicit scheme centred in space and time, the so-called box-method. From this first approach, Burges [8] improved the model by properly accounting the hydrostatic and added mass forces. These first approaches were unstable for lacking cable tension at any point of the line. Howel [9] proposed to take into account the bending stiffness of the cable to overcome the zero tension cable problems with good results. Gobat et al. [10] presented a generalized $\alpha$-method for time integration of the cable dynamics retaining the box-method for the spatial discretization.

Finite elements methods (FEM) for dynamic analysis of slender rods or mooring lines are currently wide used and validated within the offshore industry. FEM models describe the cable line as a continuous system, but the displacement field is discretized in a number of nodes. The method uses interpolation functions to describe the displacement of the internal points as well as the tangent, the curvature or the tension; and constitutive equations to describe the strain-stress relationship of the material. Three families of slender rod FEM models can be found in the literature. A first group uses linear interpolation functions to describe the cable position, [11]-[13]. These models can only take into account axial deformation and require a fine discretization of the line to accurately simulate large variations of the curvature or slack events. The second family uses larger order finite elements, from cubic to high-order polynomial elements. They allow to model properly the change of curvature of the line and also enable to take into account the bending and the torsional stiffness to improve accuracy in the slack events. They reduce the required discretization at the cost of having a larger number of nodal variables [14][17]. The third family of FEM comes from 3D beam finite element models applied for mooring lines [18] where classic 3D beam formulations are extended for large displacements and rotations, these formulations also include more variable as the spin of the element in addition to the spatial three DOF. Most of the models use variations of the Newmark's time scheme integration, like the HHT alphamethod presented by Hilber et al. [19], or the generalized- $\alpha$ method presented by Chung and Hulbert [20] which applies numerical damping dissipation to overcome numerical instabilities [21], [22].

Mooring line models use Hooke-s law constitutive equations for describing the elastic behaviour of the line in axial direction. More complex models use internal viscous damping to represent energy dissipation from axial velocity deformation due to tether friction between the layers of conductors and polymer coatings, [13], [15]. On the other hand, stranded cable damping source comes mainly from the sliding between wires during flexural vibrations [23]. The stick-slip frictional behaviour of strand cables was first described by Hardy [24] and extended by Goudreau et al. [25] which is modelled using classic Amontons-Coulomb friction law. More detailed models assess the stick-slip behaviour of each wire of the strand to account for the non-linear section bending response of the cable cross section [26]. Recently, the research in visco-elastic materials leads to new models to take into account the viscoelastic rheological damping of beams in bending, [27]-[29]. Moreover, [18] presented an extension of a beam FEM to a mooring line model that takes into account both the axial and the bending damping for mooring cables. 
In this paper, a new extension of the FEM model proposed by Garret [14] adding rheological damping material is presented. The model takes advantage of high order shape functions which describe more accurately the line shape and the addition of a visco-elastic term for accounting the damping behavior of the line due to the deformation of the element. This approach allows to assess in a more realistic way the damping contribution of the line elements due to its deformation and can be applied to chains, wire ropes and power cables. The chain elements have no bending stiffness and present higher corrosion and friction resistance than wire ropes, and are commonly used in the bottom and upper segments of the moorings lines. Wire ropes are mainly used in middle sections of long mooring lines, between chains, to reduce the total weight. Electrical wires are one of the most sensitive elements of FOWT because they ensure the connection to the grid. Then, they deserve accurate simulation to assess its performance along their service life [3].

The model is based on the Garret's formulation, a FEM model for slender rods which assumes large displacements and rotations, but small strains, by including the extensibility of the line as proposed by Kim[16]. The model is extended by accounting for internal viscous damping trough a rheological model and assessing correctly the contribution of the curvature of the rod within the strain-stress constitutive equations. The rheological damping is based on the Kelvin-Voigt model, [30], which proper defines the behavior of visco-elastic materials as used for wire and electrical cable protection and synthetic ropes [31]. The Kelvin-Voigt model is a proper model for assessing creep and relaxation which contributes for the internal damping stresses of sudden incremental loads. However, instantly stress-strain relation is not verified. Moreover, in some cases such as strand cables, the damping source is amplitude dependent instead of frequency dependent. Then, the friction damping due to the stick-slip phenomenon has to be considered as an equivalent viscous damping evaluated for an estimated critical damping ratio, which no fulfils exactly the physical phenomenon [32]. However, the use of a rheological model is more computationally efficient compared with the cross section fiber damping analysis. This rheological model is applied to the axial and the bending internal forces. Derivation of the axial and bending damping forces are presented and assessed in terms of the critical damping ratio for both forces. The curvature of the rod is precisely included by a Lagrange multiplier, which relates the tension and the derivative of the moment. This Lagrange multiplier is included in the strain-stress constitutive equation to combine correctly the equations of motion and deformation. The use of the Lagrange multiplier implies the addition of a new variable, thus the number of degrees of freedom of the problem is also increased. Otherwise, the main advantage is the direct relation of the stress-strain relation equation with the equations of motions of the slender rod. Then, both system of equations can be solved jointly in a larger system of equations in a matrix way. In the other case, the internal stress would be an explicit term and should be assessed at the end of each iteration. Using the internal stresses as an explicit term would increase the number of iterations to get a proper tolerance of the solution.

The paper presents the differential equations of motion of the slender rods, the finite element integration, the initial static configuration and the time integration schemes of the proposed model.

The accuracy of the model is appraised with three examples of verification followed by two validation cases. The verification analyses consist in the numerical simulation of the vibration of a rod in axial and bending forces with different values of the critical damping. The validation examples are more complex and consist in, first, comparing the experimental results of the free fall of a dynamic cable presented by Koh [33] and, second, the simulation of the experimental results of a mooring chain with a cyclic imposed motion at one end [11], [34]. 


\section{Rheological damping material}

The rheological behaviour of viscoelastic solids may be described by the Kelvin-Voigt model which includes a spring and a dashpot in parallel as depicted in Figure 1, [30]. The strain-stress relation of the material for a constant initial stress presents a creep behavior transforming all stress to the spring at infinite time. However, instant stress-train relation behavior is not presented as certain time is needed to achieve the elastic tension. This time is related with the retardation time.

The time retardation is the ratio between $\eta / E$ [35] and in the cases dealt in this paper is about 1 and 4 $\%$ of the vibration period.

The differential equation that defines the strain-stress behavior can be expressed by Eq. (1), where $\sigma$ is the normal tension of the cross section of the line, $\varepsilon$ is the axial strain of a line section, $E$ and $\eta$ are the Young modulus and the material viscosity respectively. In all the following development, direct proportion between stress and strains is assumed (Hooke's law).

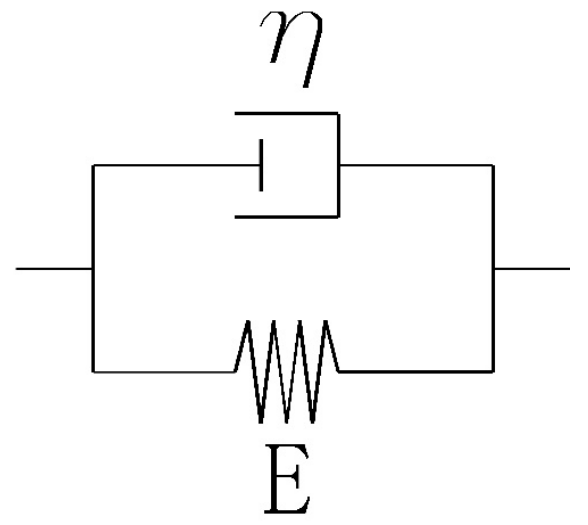

Figure 1: Kelvin-Voigt rheological model

$$
\sigma=\mathrm{E} \varepsilon+\eta \frac{\partial \varepsilon}{\partial t}
$$

Then, in absence of bending, the axial force is obtained directly by integrating the uniform axial stress:

$$
\mathrm{N}=\int \sigma \mathrm{dA}=\mathrm{EA} \varepsilon+\eta \mathrm{A} \frac{\partial \varepsilon}{\partial t}
$$

From Eq. (1), the internal bending moment can be obtained by the integration of the stress multiplied by the distance to the neutral axis of the cross section, in this case the centerline. Assuming the EulerBernoulli beam approach, the strain-curvature relation can be expressed as $\varepsilon \cong \kappa \cdot z$, where $z$ is the transverse distance with origin at the neutral axis and normal to axial coordinate in the osculating plane, and $\kappa$ is the cable curvature. Then, the curvature velocity can be approximate from the axial strain velocity as follows $\frac{\partial \varepsilon}{\partial t} \cong \frac{\partial \kappa}{\partial t} z$. Thus, the moment is expressed in Eq. (3), where $I$ is the second moment of area of the cross section.

$$
\mathrm{M}=\int \sigma \cdot \mathrm{zdA}=\int\left(\mathrm{E} \varepsilon+\eta \frac{\partial \varepsilon}{\partial t}\right) \cdot z d A=\int\left(\mathrm{E} \kappa+\eta \frac{\partial \kappa}{\partial \mathrm{t}}\right) \cdot z^{2} d A=I\left(\mathrm{E} \kappa+\eta \frac{\partial \kappa}{\partial t}\right)
$$




\section{Rod model adding rheological damping}

The adopted rod model is a FEM model based on the formulation presented by Garret [14] which is derived from the translational and rotatory equations of motions of a slender rod for large displacements and rotations. Also the material stiffness and the small strain assumption is accounted as proposed by Kim [16]. The model is extended to apply the material damping for both axial and bending deformations, the normal strains.

The cable is defined by the centreline position $\mathbf{r}(s, t)$ as a function of the arc-length parameter " $s$ " and the time " $t$ ", as is shown in Figure 2. The centreline position defines the deformed shape of the line. At any point of the line the tangent vector of the curve $\mathbf{t}$ is defined as the unit vector of the derivative of the centreline position respect to the arc-length parameter, where the norm of the derivative of the centreline is equal to the strain plus the unit. The normal vector $\mathbf{n}$ is defined as the unit vector of the derivative of the tangent vector, and can also be expressed as the second derivative of the line position divided by the curvature $(k)$. The tangent and the normal vectors define the osculating plane at each point. The binormal vector $\mathbf{b}$ is defined as the cross product of $\mathbf{t}$ and $\mathbf{n}$. The tangent, normal and binormal vectors are defined in Eq. (4), where prime denotes differentiation respect to "s". The global coordinates is such as the $z$ direction is pointing against the gravity. The unitary global vectors are defined as $\left[\boldsymbol{e}_{\boldsymbol{x}}, \boldsymbol{e}_{\boldsymbol{y}}, \boldsymbol{e}_{z}\right]$.

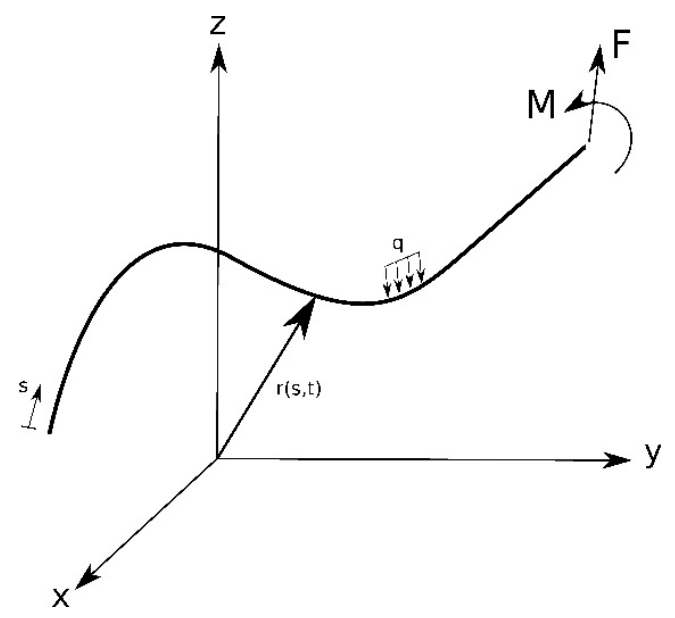

Figure 2: cable centerline sketch

$$
\mathbf{t}=\frac{\partial \mathbf{r} / \partial s}{\|\partial \mathbf{r} / \partial s\|}=\frac{\mathbf{r}^{\prime}}{\|\mathbf{r} \prime\|} ; \mathbf{n}=\frac{1}{\kappa} \frac{d \mathbf{t}}{d s}=\frac{1}{\kappa} \frac{\mathbf{r} \prime \prime}{\|\mathbf{r} /\|} ; \mathbf{b}=\mathbf{t} \times \mathbf{n}
$$

where:

$\left\|\mathbf{r}^{\prime}\right\|=(1+\varepsilon), \varepsilon$ is the strain, and $\kappa$ is the curvature and is defined as $\kappa=\frac{\left\|\mathbf{r}^{\prime \prime}\right\|}{\left\|\mathbf{r}^{\prime}\right\|}$

\subsection{Derivation of the equation of motion}

Conservation of the linear and angular momenta of a differential cable element leads to the translational equation of motion Eq. (5) and to the static rotatory equation of motion Eq. (6) by neglecting the rotatory inertia and the shear deformation. $\mathbf{F}$ and $\mathbf{M}$ are the resultant force and moment respectively of the internal stress state at one point acting on the centreline, $\mathbf{q}$ and $\mathbf{m}$ are the external 
applied force and moment per unit length, $\rho_{m}$ is the line density, $A$ is the cross section of the line, and the superposed dot denotes differentiation with respect to time.

$$
\begin{aligned}
& \mathbf{F}^{\prime}+\mathbf{q}=\rho_{m} A \ddot{\mathbf{r}} \\
& \mathbf{M}^{\prime}+\frac{\mathbf{r}^{\prime}}{\left\|\mathbf{r}^{\prime}\right\|} \times \mathbf{F}+\mathbf{m}=0
\end{aligned}
$$

The resultant moment for an elastic rod according to Euler-Bernoulli theory with equal principal stiffness, adding rheological damping material and disregarding the torsional component of the stress is expressed in Eq. (7). This equation is derived from the moment Eq. (3) in terms of $\mathbf{t}$ and $\mathbf{n}$.

$$
\begin{aligned}
& \mathbf{M}=E I \kappa \mathbf{b}+\eta I \dot{\kappa} \mathbf{b}=\frac{\mathbf{r}^{\prime}}{\left\|\mathbf{r}^{\prime}\right\|} \times\left(\left(E I+\eta I \frac{\dot{\kappa}}{\kappa}\right) \frac{\mathbf{r}^{\prime \prime}}{\left\|\mathbf{r}^{\prime}\right\|}\right)=\frac{\mathbf{r}^{\prime}}{\|\mathbf{r} \prime\|} \times\left(E I \frac{\mathbf{r}^{\prime \prime}}{\left\|\mathbf{r}^{\prime}\right\|}+\eta I\left(\frac{\mathbf{r}^{\prime \prime} \cdot \dot{\mathbf{r}}^{\prime \prime}}{\mathbf{r}^{\prime \prime} \cdot \mathbf{r}^{\prime \prime}}-\right.\right. \\
& \left.\left.\frac{\mathbf{r}^{\prime} \cdot \dot{\mathbf{r}}^{\prime}}{\mathbf{r}^{\prime} \cdot \mathbf{r}^{\prime}}\right) \frac{\mathbf{r}^{\prime \prime}}{\left\|\mathbf{r}^{\prime}\right\|}\right)
\end{aligned}
$$

The curvature velocity $(\dot{\kappa})$ is obtained by the derivative of the curvature respect to time, as is shown in Eq. (8). The equation states the relation between the curvature velocity and the difference between the normal and tangent velocities. In a simplified way, it means that if the velocity of the normal vector increases in a given proportion with the tangent velocity, the curvature remains constant and then, the curvature velocity is zero.

$$
\dot{\kappa}=\frac{\left(\mathbf{r}^{\prime \prime} \cdot \dot{\mathbf{r}}^{\prime \prime}\right)}{\left(\mathbf{r}^{\prime} \cdot \mathbf{r}^{\prime}\right)^{1 / 2} \cdot\left(\mathbf{r}^{\prime \prime} \cdot \mathbf{r}^{\prime \prime}\right)^{1 / 2}}-\frac{\left(\mathbf{r}^{\prime \prime} \cdot \mathbf{r}^{\prime \prime}\right)^{1 / 2} \cdot\left(\mathbf{r}^{\prime} \cdot \dot{\mathbf{r}}^{\prime}\right)}{\left(\mathbf{r}^{\prime} \cdot \mathbf{r}^{\prime}\right)^{3 / 2}}
$$

Then, the ratio of the curvature velocity respect to the curvature found in Eq. (7) can be expressed as:

$$
\frac{\dot{\kappa}}{\kappa}=\frac{\mathbf{r}^{\prime \prime} \cdot \dot{\mathbf{r}}^{\prime \prime}}{\mathbf{r}^{\prime \prime} \cdot \mathbf{r}^{\prime \prime}}-\frac{\mathbf{r}^{\prime} \cdot \dot{\mathbf{r}}^{\prime}}{\mathbf{r}^{\prime} \cdot \mathbf{r}^{\prime}}
$$

In order to expand the Eq. (6), the derivative of the resultant moment from Eq. (7) respect to the arc length has the form of:

$$
\mathbf{M}^{\prime}=\frac{\mathbf{r}^{\prime}}{\left\|\mathbf{r}^{\prime}\right\|} \times\left(E I \frac{\mathbf{r}^{\prime \prime}}{\|\mathbf{r} \prime\|}+\eta I\left(\frac{\mathbf{r}^{\prime \prime} \cdot \dot{\mathbf{r}}^{\prime \prime}}{\mathbf{r}^{\prime \prime} \cdot \mathbf{r}^{\prime \prime}}-\frac{\mathbf{r}^{\prime} \cdot \dot{\mathbf{r}}^{\prime}}{\mathbf{r}^{\prime} \cdot \mathbf{r}^{\prime}}\right) \frac{\mathbf{r}^{\prime \prime}}{\|\mathbf{r} \mathbf{}\|}\right)^{\prime}
$$

Next, substituting Eq. (10) into Eq. (6) and neglecting the applied linear moment per unit length $\mathbf{m}$ leads to:

$$
\frac{\mathbf{r}^{\prime}}{\left\|\mathbf{r}^{\prime}\right\|} \times\left(\left(E I \frac{\mathbf{r}^{\prime \prime}}{\|\mathbf{r} \prime\|}+\eta I\left(\frac{\mathbf{r}^{\prime \prime} \cdot \dot{\mathbf{r}}^{\prime \prime}}{\mathbf{r}^{\prime \prime} \cdot \mathbf{r}^{\prime \prime}}-\frac{\mathbf{r}^{\prime} \cdot \dot{\mathbf{r}}^{\prime}}{\mathbf{r}^{\prime} \cdot \mathbf{r}^{\prime}}\right) \frac{\mathbf{r}^{\prime \prime}}{\left\|\mathbf{r}{ }^{\prime}\right\|}\right)^{\prime}+\mathbf{F}\right)=0
$$

This Eq. (11) shows the relationship between $\mathbf{r}, \mathbf{F}$ and $\mathbf{M}^{\prime}$. Then, $\mathbf{F}$, and $\mathbf{M}^{\prime}$ must be proportional to the tangent vector $\mathbf{t}$, and this relationship can be expressed as follows.

$$
\left(E I \frac{\mathbf{r}^{\prime \prime}}{\|\mathbf{r} \prime\|}+\eta I\left(\frac{\mathbf{r}^{\prime \prime} \cdot \dot{\mathbf{r}}^{\prime \prime}}{\mathbf{r}^{\prime \prime} \cdot \mathbf{r}^{\prime \prime}}-\frac{\mathbf{r}^{\prime} \cdot \dot{\mathbf{r}}^{\prime}}{\mathbf{r}^{\prime} \cdot \mathbf{r}^{\prime}}\right) \frac{\mathbf{r}^{\prime \prime}}{\left\|\mathbf{r}{ }^{\prime}\right\|}\right)^{\prime}+\mathbf{F}=\lambda \frac{\mathbf{r}^{\prime}}{\left\|\mathbf{r}^{\prime}\right\|}
$$

Where $\lambda(s, t)$ is a scalar, and can be identified as the Lagrange multiplier. Then, the force $\mathbf{F}$ is of the form of:

$$
\mathbf{F}=-\left(E I \frac{\mathbf{r}^{\prime \prime}}{\|\mathbf{r} \prime\|}+\eta I\left(\frac{\mathbf{r}^{\prime \prime} \cdot \dot{\mathbf{r}}^{\prime \prime}}{\mathbf{r}^{\prime \prime} \cdot \mathbf{r}^{\prime \prime}}-\frac{\mathbf{r}^{\prime} \cdot \dot{\mathbf{r}}^{\prime}}{\mathbf{r}^{\prime} \cdot \mathbf{r}^{\prime}}\right) \frac{\mathbf{r}^{\prime \prime}}{\left\|\mathbf{r}^{\prime}\right\|}\right)^{\prime}+\lambda \frac{\mathbf{r}^{\prime}}{\left\|\mathbf{r}^{\prime}\right\|}
$$

The scalar product of Eq. (13) with $\frac{\mathbf{r}^{\prime}}{\left\|\mathbf{r}^{\prime}\right\|}$ leads to an expression that defines the Lagrange multiplier: 


$$
\lambda=T-\left(E I+\eta I\left(\frac{\mathbf{r}^{\prime \prime} \cdot \dot{\mathbf{r}}^{\prime \prime}}{\mathbf{r}^{\prime \prime} \cdot \mathbf{r}^{\prime \prime}}-\frac{\mathbf{r}^{\prime} \cdot \dot{\mathbf{r}}^{\prime}}{\mathbf{r}^{\prime} \cdot \mathbf{r}^{\prime}}\right)\right) \kappa^{2}
$$

Since:

$$
\begin{gathered}
\left\{\begin{array}{c}
\lambda \frac{\mathbf{r}^{\prime}}{\left\|\mathbf{r}^{\prime}\right\|} \cdot \frac{\mathbf{r}^{\prime}}{\left\|\mathbf{r}^{\prime}\right\|}=\lambda \frac{\left\|\mathbf{r}^{\prime}\right\|^{2}}{\left\|\mathbf{r}^{\prime}\right\|^{2}}=\lambda \\
\mathbf{F} \frac{\mathbf{r}^{\prime}}{\left\|\mathbf{r}^{\prime}\right\|}=\mathbf{F} \cdot \mathbf{t}=\mathbf{T} \\
\left(X \frac{\mathbf{r}^{\prime \prime}}{\left\|\mathbf{r}^{\prime}\right\|}\right)^{\prime} \frac{\mathbf{r}^{\prime}}{\left\|\mathbf{r}^{\prime}\right\|}=\left(E I \frac{\mathbf{r}^{\prime \prime}}{\left\|\mathbf{r}^{\prime}\right\|} \cdot \frac{\mathbf{r}^{\prime}}{\left\|\mathbf{r}^{\prime}\right\|}\right)^{\prime}-X \frac{\mathbf{r}^{\prime \prime}}{\left\|\mathbf{r}^{\prime}\right\|} \cdot\left(\frac{\mathbf{r}^{\prime}}{\left\|\mathbf{r}^{\prime}\right\|}\right)^{\prime}=-X \frac{\mathbf{r}^{\prime \prime}}{\left\|\mathbf{r}^{\prime}\right\|} \cdot \frac{\mathbf{r}^{\prime \prime}}{\left\|\mathbf{r}^{\prime}\right\|}=-X \frac{\left\|\mathbf{r}^{\prime \prime}\right\|^{2}}{\left\|\mathbf{r}^{\prime}\right\|^{2}}=-X \kappa^{2}
\end{array}\right. \\
\text { Where } X=E I+\eta_{B} I\left(\frac{\mathbf{r}^{\prime \prime} \cdot \dot{\mathbf{r}}^{\prime \prime}}{\mathbf{r}^{\prime \prime} \cdot \mathbf{r}^{\prime \prime}}-\frac{\mathbf{r}^{\prime} \cdot \dot{\mathbf{r}}^{\prime}}{\mathbf{r}^{\prime} \cdot \mathbf{r}^{\prime}}\right)
\end{gathered}
$$

If the Eq. (13) is substituted in the Eq. (5) the following translational equation of motion is obtained:

$$
-\left(E I \frac{\mathbf{r}^{\prime \prime}}{\|\mathbf{r} \prime\|}+\eta I\left(\frac{\mathbf{r}^{\prime \prime} \cdot \dot{\mathbf{r}}^{\prime \prime}}{\mathbf{r}^{\prime \prime} \cdot \mathbf{r}^{\prime \prime}}-\frac{\mathbf{r}^{\prime} \cdot \dot{\mathbf{r}}^{\prime}}{\mathbf{r}^{\prime} \cdot \mathbf{r}^{\prime}}\right) \frac{\mathbf{r}^{\prime \prime}}{\left\|\mathbf{r}{ }^{\prime}\right\|}\right)^{\prime \prime}+\left(\lambda \frac{\mathbf{r}^{\prime}}{\left\|\mathbf{r}^{\prime}\right\|}\right)^{\prime}+\mathbf{q}=\rho_{m} A \ddot{\mathbf{r}}
$$

\subsection{Inclusion of the constitutive equation}

The constitutive equation of a rod relates the axial strain with the tension. The derivative of the position of the rod is equal to the unity plus the strain, $\left\|\mathbf{r}^{\prime}\right\|=1+\varepsilon$. Then, if the stretch of the rod is assumed to be linear and small, then the next equation is obtained and further approximated as:

$$
\mathbf{r}^{\prime} \cdot \mathbf{r}^{\prime}=(1+\varepsilon)^{2}=1+2 \varepsilon+O\left(\varepsilon^{2}\right)
$$

Then, isolating $\varepsilon$ we obtain:

$$
\varepsilon=\frac{1}{2}\left(\mathbf{r}^{\prime} \cdot \mathbf{r}^{\prime}-1\right)
$$

The relation between tension and strain for each element is described in Eq. (18) from the internal axial force of Eq. (2).

$$
T=N=A E \varepsilon+\eta A \dot{\varepsilon}
$$

From the strain $(\varepsilon)$ defined in Eq. (17), the following approximation of the strain velocity by the derivative respect to time is obtained:

$$
\dot{\varepsilon}=\frac{\partial}{\partial t} \varepsilon=\frac{\partial}{\partial t}\left(\frac{1}{2}\left(\mathbf{r}^{\prime} \cdot \mathbf{r}^{\prime}-1\right)\right)=\mathbf{r}^{\prime} \cdot \dot{\mathbf{r}}^{\prime}
$$

Then, substituting Eq. (17) and Eq. (19) into Eq. (18), the Eq. (14) can be expressed as follows, which relates the strain with the Lagrange multiplier:

$$
0=\frac{1}{2}\left(\mathbf{r}^{\prime} \cdot \mathbf{r}^{\prime}-1\right)+\frac{\eta}{E}\left(\mathbf{r}^{\prime} \cdot \dot{\mathbf{r}}^{\prime}\right)-\left(\frac{E I}{E A}+\frac{\eta I}{E A}\left(\frac{\mathbf{r}^{\prime \prime} \cdot \dot{\mathbf{r}}^{\prime \prime}}{\mathbf{r}^{\prime \prime} \cdot \mathbf{r}^{\prime \prime}}-\frac{\mathbf{r}^{\prime} \cdot \dot{\mathbf{r}}^{\prime}}{\mathbf{r}^{\prime} \cdot \mathbf{r}^{\prime}}\right)\right) \kappa^{2}-\frac{\lambda}{E A}
$$

The dynamics of a slender rod with rheological damping material is fully described by Eq. (15) and Eq. (20). The Eq. (15) is the equation of motion of the centreline and the Eq. (20) fulfils the constitutive equations relating the strain and the curvature of the line to its equation of motion by the Lagrange multiplier. 


\subsection{Critical Damping assessment}

The damping term (c) of the dynamic equation of a vibrating system, Eq. (21), is usually characterized by critical damping coefficient $\left(c_{c r}\right)$ through the damping ratio $\xi$ by $c=\xi \cdot c_{c r}$. The critical damping coefficient and the natural frequency is defined by Eq. (22) and Eq. (23) respectively [36].

$$
\begin{aligned}
& m \ddot{x}(t)+c \dot{x}(t)+k x(t)=0 \\
& c_{c r}=2 \sqrt{k m} \\
& \omega_{d}=\omega_{n} \sqrt{1-\xi^{2}}, \text { where } \omega_{n}=\sqrt{k / m}
\end{aligned}
$$

The critical viscosity, which implies a critical damping behavior for each force, are derived in the next equations for the axial force and the bending moment. This characterization is obtained assuming that the rod is only subjected to a pure axial load or pure bending moment in each case. This procedure allows to assess the viscosity of a loaded rod with its boundary conditions for a given damping ratio. Then, using a simple vibration test of a rod and assessing its damping behavior the related viscosity can be obtained.

For a better comprehension of the following partial differential equations of next sections, derivatives respect to the time and to the rod axial direction are expressed by subscripts $t$ and $x$ respectively.

\subsubsection{Axial Critical Damping}

Considering only the elongation of a rod without any other external force excluded the axial force, the translational equation of motion Eq. (5) can be reformulated in terms of the displacement of the rod $(u)$ and the Kelvin-Voigt rheological model for the axial stress-strain relation is expressed as Eq. (24). The strain as a function of the rod axial displacement is expressed as $\varepsilon=u_{x}$.

$$
\begin{aligned}
& \rho_{m} A \frac{\partial^{2} u}{\partial t^{2}}-\frac{\partial(T)}{\partial x}=\rho_{m} A \frac{\partial^{2} u}{\partial t^{2}}-\frac{\partial\left(E A \varepsilon+\eta A \varepsilon_{t}\right)}{\partial x}=\rho_{m} A u_{t t}-A E u_{x x}-\eta A u_{x x t}=\rho_{m} u_{t t}- \\
& E u_{x x}-\eta u_{x x t}=0
\end{aligned}
$$

Assuming the separation of variables $(x, t)=\phi(x) h(t)$, Eq. (24) yields in:

$$
\rho_{m} \phi h_{t t}-E h \phi_{x x}-\eta h_{t} \phi_{x x}=0
$$

Eq. (25) can be rewritten in Eq. (26), where the right side depends of $t$ and the left side of $x$. Then, the unique solution is a scalar defined as $-\beta_{A}^{2},[36]$.

$$
\frac{\rho_{m}}{\left(\eta h_{t}+E h\right)} h_{t t}=\frac{1}{\phi} \phi_{x x}=-\beta_{\mathrm{A}}^{2}
$$

Then, the left side of Eq. (26) can be rewritten as a temporal ordinary differential equation (ODE) as shown in Eq. (27).

$$
\rho_{m} h_{t t}+\eta \beta_{A}^{2} h_{t}+E \beta_{A}^{2} h=0
$$

Then, the critical damping behavior of an axially deformed rod is produced by the critical viscosity:

$$
\eta_{A, \text { Critic }}=2 / \beta_{A} \sqrt{\rho_{m} \cdot E}
$$

For a cantilever rod with a free end motion, $\beta_{A}$ can be assessed from the next boundary conditions: no motion of the fixed point, $u(0, t)=0$, and no tension on the free end, thus $u_{x}(L, t)=0$. Then, applying 
these assumptions to the ODE of the left side of Eq. (26), $\phi_{x x}+\beta_{A}^{2} \phi=0$, the mode shapes obtained of the rod are $\beta_{A, n}=\left(n \pi-\frac{\pi}{2}\right) / L$, whereby the first mode is $\beta_{A, 1}=\frac{\pi}{2 L}$.

\subsubsection{Bending Critical Damping}

Following the same procedure as for the axial critical damping, the Eq. (29) represents the partial differential equation of motion the transverse vibration due to a bending moment of an Euler-Bernoulli beam.

$$
\rho_{m} A \frac{\partial^{2} \omega}{\partial t^{2}}+\frac{\partial^{2}}{\partial x^{2}}\left(E I \frac{\partial^{2} \omega}{\partial x^{2}}+\eta I \frac{\partial^{3} \omega}{\partial x^{2} \partial t}\right)=\rho_{m} \frac{\partial^{2} \omega}{\partial t^{2}}+E I \frac{\partial^{4} \omega}{\partial x^{4}}+\eta I \frac{\partial^{5} \omega}{\partial x^{4} \partial t}=0
$$

Assuming again the separation of variables, $\omega$ can be defined as a product $\omega(x, t)=\phi(x) h(t)$, then:

$$
\rho_{m} A \phi h_{t t}+E I h \phi_{x x x x}+\eta I h_{t} \phi_{x x x x}=0
$$

In Eq. (30), there are different terms depending on $t$ and on $x$, then, the unique solution is a scalar defined as $-\beta_{B}^{4}$.

$$
\frac{\rho_{m} A}{\left(\eta h_{t}+E I h\right)} h_{t t}=-\frac{1}{\phi} \phi_{x x x x}=-\beta_{B}^{4}
$$

Considering the left part of Eq. (31), the equation can be rewritten as an ODE as shown in Eq. (32).

$$
\rho_{m} A h_{t t}+\eta I \beta_{B}^{4} h_{t}+E I \beta_{B}^{4} h=0
$$

Then, the critical damping behavior of a bending deformed rod is achieved by the critical viscosity:

$$
\eta_{B, \text { Critic }}=2 / \beta_{B}^{2} \sqrt{\rho_{m} A \cdot E / I}
$$

For a cantilever beam with a free end motion, the $\beta_{A}$ can be assessed from the next boundary conditions: no deflection and straight direction of the bam at the fixed point, $\omega(0, t)=0$ and $\frac{\partial \omega}{\partial x}(0, t)=0$, and no moment and no shearing force at the free end, $\frac{\partial^{2} \omega}{\partial x^{2}}(L, t)=0$ and $\frac{\partial^{3} \omega}{\partial x^{3}}(L, t)=0$. Then, applying these assumptions to the ODE $\phi_{x x x x}-\beta_{B}^{4} \phi=0$, the first bending mode is expressed as $\beta_{B, 1}=0.59686 \pi / L,[36]$.

\section{Finite Element Method implementation}

The Eq. (15) and Eq. (20) that represent the dynamics of the system including the constitutive behaviour in axial and bending, respectively, are unified in Eq. (34). Both equations will be treated jointly to apply the finite element Garlekin method.

$$
\left.\begin{array}{c}
\rho_{m} A \ddot{\mathbf{r}}+\left(E I \frac{\mathbf{r}^{\prime \prime}}{\left\|\mathbf{r}^{\prime}\right\|}+\eta_{B} I\left(\frac{\mathbf{r}^{\prime \prime} \cdot \dot{\mathbf{r}}^{\prime \prime}}{\mathbf{r}^{\prime \prime} \cdot \mathbf{r}^{\prime \prime}}-\frac{\mathbf{r}^{\prime} \cdot \dot{\mathbf{r}}^{\prime}}{\mathbf{r}^{\prime} \cdot \mathbf{r}^{\prime}}\right) \frac{\mathbf{r}^{\prime \prime}}{\left\|\mathbf{r}^{\prime}\right\|}\right)^{\prime \prime}-\left(\lambda \frac{\mathbf{r}^{\prime}}{\left\|\mathbf{r}^{\prime}\right\|}\right)^{\prime}=\mathbf{q} \\
\frac{1}{2}\left(\mathbf{r}^{\prime} \cdot \mathbf{r}^{\prime}-1\right)+\frac{\eta_{A}}{E}\left(\mathbf{r}^{\prime} \cdot \dot{\mathbf{r}}^{\prime}\right)-\left(\frac{E I}{E A}+\frac{\eta_{B} I}{E A}\left(\frac{\mathbf{r}^{\prime \prime} \cdot \dot{\mathbf{r}}^{\prime \prime}}{\mathbf{r}^{\prime \prime} \cdot \mathbf{r}^{\prime \prime}}-\frac{\mathbf{r}^{\prime} \cdot \dot{\mathbf{r}}^{\prime}}{\mathbf{r}^{\prime} \cdot \mathbf{r}^{\prime}}\right)\right) \kappa^{2}-\frac{\lambda}{E A}=0
\end{array}\right\}
$$

Consider a single element of length $L$, the position of the centreline of the rod element $(r)$, and the Lagrange multiplier $(\lambda)$ are expressed as the sum of the approximate values of the nodes multiplied by its shape functions as is shown in Eq. (35) and, Eq. (36) where $N_{l}, P_{m}$ are the shape functions, and $U_{i l}, \lambda_{m}$ are the unknown coefficients, where $0 \leq s \leq L$ and the subscript $i=1,2,3$ that denotes the 3 DOF of the node position. 


$$
\begin{aligned}
& r_{i}(s, t)=\sum_{l} N_{l}(s) U_{i l}(t) \\
& \lambda(s, t)=\sum_{m} P_{m}(s) \lambda_{m}(t)
\end{aligned}
$$

The Hermite interpolation shape functions are used to define the rod position vector $\left(N_{l}\right)$, and the quadratic shape functions are used to interpolate the Lagrange multiplier $\left(P_{m}\right)$, shown in Eq. (37) where $\xi=\frac{s}{L}$.

$$
\left.\begin{array}{c}
N_{1}=1-3 \xi^{2}+2 \xi^{3} \\
N_{2}=d l\left(\xi-2 \xi^{2}+\xi^{3}\right) \\
N_{3}=3 \xi^{2}-2 \xi^{3} \\
N_{4}=d l\left(-\xi^{2}+\xi^{3}\right)
\end{array}\right\}
$$

Then, the position $\left(r_{i}\right)$, the tangent $\left(r_{i}^{\prime}\right)$ and the Lagrange multiplier $(\lambda)$ are evaluated at each node. The parameters $U_{i l}$ and $\lambda_{m}$ are thus:

$$
\begin{aligned}
& U_{i 1}=r_{i}\left(s_{i}, t\right) \\
& U_{i 2}=r_{i}^{\prime}\left(s_{i}, t\right) \\
& U_{i 3}=r_{i}\left(s_{i+1}, t\right) \\
& U_{i 4}=r_{i}^{\prime}\left(s_{i+1}, t\right) \\
& \lambda_{1}=\lambda\left(s_{i}\right) \\
& \lambda_{2}=\lambda\left(s_{i}+d l / 2\right) \\
& \lambda_{3}=\lambda\left(s_{i+1}\right)
\end{aligned}
$$

Applying the Garlekin method to the weak form of the Eq. (34) by multiplying the shape function by its respective equation, the Eq. (39) is obtained.

$$
\left.\begin{array}{c}
\int_{0}^{L} N_{l}\left(\rho_{m} A \ddot{\boldsymbol{r}}+\left(E I \frac{\boldsymbol{r}^{\prime \prime}}{\left\|\boldsymbol{r}^{\prime}\right\|}+\eta_{B} I\left(\frac{\boldsymbol{r}^{\prime \prime} \cdot \dot{\boldsymbol{r}}^{\prime \prime}}{\boldsymbol{r}^{\prime \prime} \cdot \boldsymbol{r}^{\prime \prime}}-\frac{\boldsymbol{r}^{\prime} \cdot \dot{\boldsymbol{r}}^{\prime}}{\boldsymbol{r}^{\prime} \cdot \boldsymbol{r}^{\prime}}\right) \frac{\boldsymbol{r}^{\prime \prime}}{\left\|\boldsymbol{r}^{\prime}\right\|}\right)^{\prime \prime}-\left(\lambda \frac{\boldsymbol{r}^{\prime}}{\left\|\boldsymbol{r}^{\prime}\right\|}\right)^{\prime}-\boldsymbol{q}\right) d s=0 \\
\int_{0}^{L} P_{m}\left(\frac{1}{2}\left(\boldsymbol{r}^{\prime} \cdot \boldsymbol{r}^{\prime}-1\right)+\frac{\eta_{A}}{E}\left(\boldsymbol{r}^{\prime} \cdot \dot{\boldsymbol{r}}^{\prime}\right)-\left(\frac{E I}{E A}+\frac{\eta_{B} I}{E A}\left(\frac{\boldsymbol{r}^{\prime \prime} \cdot \dot{\boldsymbol{r}}^{\prime \prime}}{\boldsymbol{r}^{\prime \prime} \cdot \boldsymbol{r}^{\prime \prime}}-\frac{\boldsymbol{r}^{\prime} \cdot \dot{\boldsymbol{r}}^{\prime}}{\boldsymbol{r}^{\prime} \cdot \boldsymbol{r}^{\prime}}\right)\right) \kappa^{2}-\frac{\lambda}{E A}\right) d s=0
\end{array}\right\}
$$

The integration of the Eq. (39) leads to the dynamic system of equations expressed in Eq. (40):

$$
\left.\begin{array}{c}
M_{i j l k} \ddot{U}_{j k}+\left(K_{i j l k}^{1}+K_{i j l k}^{1, D a m p}+\lambda_{n} K_{n i j l k}^{2}\right) U_{j k}-F_{i l}^{e}=0 \\
A_{m i l} U_{j l} U_{j k}-B_{m}+D_{m n} U_{k l} \dot{U}_{l k}-E_{m i l}(U, \dot{U})-C_{m n} \lambda_{n}=0
\end{array}\right\}
$$

where:

$$
\begin{aligned}
& M_{i j l k}=\int_{0}^{L} \rho N_{l} N_{k} \delta_{i j} d s \\
& K_{i j l k}^{1}=\int_{0}^{L} \frac{E I N_{l}^{\prime \prime} N_{k}^{\prime \prime}}{\sqrt{\left(N_{s}^{\prime} U_{j s}\right)^{T} \cdot N_{t}^{\prime} U_{j t}}} \delta_{i j} d s \\
& K_{i j l k}^{1, \text { Damp }}=\int_{0}^{L} \eta_{B} I\left(\frac{\left(N_{s}^{\prime \prime} U_{j s}\right)^{T} \cdot N_{t}^{\prime} \dot{U}_{i t}}{\left(N_{s}^{\prime \prime} U_{j s}\right)^{T} \cdot N_{t}^{\prime \prime} U_{i t}}-\frac{\left(N_{s}^{\prime} U_{j s}\right)^{T} \cdot N_{t}^{\prime} \dot{U}_{i t}}{\left(N_{s}^{\prime} U_{j s}\right)^{T} \cdot N_{t}^{\prime} U_{i t}}\right) \frac{N_{l}^{\prime \prime} N_{k}^{\prime \prime}}{\sqrt{\left(N_{s}^{\prime} U_{j s}\right)^{T} \cdot N_{t}^{\prime} U_{i t}}} \delta_{i j} d s
\end{aligned}
$$




$$
\begin{aligned}
& K_{n i j l k}^{2}=\int_{0}^{L} P_{n} N_{l}^{\prime} N_{k}^{\prime} \frac{1}{\sqrt{\left(N_{s}^{\prime} U_{j s}\right)^{T} \cdot N_{t}^{\prime} U_{j t}}} \delta_{i j} d s \\
& F_{i l}^{e}=\int_{0}^{L} N_{l} \mathbf{q} d s \\
& A_{m i l}=\frac{1}{2} \int_{0}^{L} P_{n} N_{l}^{\prime} N_{k}^{\prime} d s \\
& B_{m}=\frac{1}{2} \int_{0}^{L} P_{m} d s \\
& C_{m n}=\frac{1}{E A} \int_{0}^{L} P_{m} P_{n} d s \\
& D_{m i l}=\frac{\eta_{A}}{E} \int_{0}^{L} P_{m} N_{l}^{\prime} N_{k}^{\prime} d s \\
& E_{m i l}(U, \dot{U})=\frac{E I}{E A} \int_{0}^{L} P_{m} \frac{\left(N_{s}^{\prime \prime} U_{j s}\right)^{T} \cdot N_{t}^{\prime \prime} U_{i t}}{\left(N_{s}^{\prime} U_{j s}\right)^{T} \cdot N_{t}^{\prime} U_{i t}} d s+\frac{\eta_{B} I}{E A} \int_{0}^{L} P_{m}\left(\frac{\left(N_{s}^{\prime \prime} U_{j s}\right)^{T} \cdot N_{t}^{\prime} \dot{U}_{i t}}{\left(N_{s}^{\prime \prime} U_{j s}\right)^{T} \cdot N_{t}^{\prime \prime} U_{i t}}-\right. \\
& \left.\frac{\left(N_{s}^{\prime} U_{j s}\right)^{T} \cdot N_{t}^{\prime} \dot{U}_{i t}}{\left(N_{s}^{\prime} U_{j s}\right)^{T} \cdot N_{t}^{\prime} U_{i t}}\right) \frac{\left(N_{s}^{\prime \prime} U_{j s}\right)^{T} \cdot N_{t}^{\prime \prime} U_{i t}}{\left(N_{s}^{\prime} U_{j s}\right)^{T} \cdot N_{t}^{\prime} U_{i t}} d s
\end{aligned}
$$

where $\delta_{i j}$ is the Kronecker delta which ensures the independence between the DOF of the orthonormal basis of $\mathbb{R}^{3}$.

\section{Static problem}

The initial problem of the reference configuration of the line shape is solved from the static point of view of the FEM formulation presented in section 4. Thus, the rod is supposed to be loaded from external forces such as the weight, but node accelerations and velocities are set to zero. Then, the dynamic system of equations can be expressed as Eq. (51), from an $n^{\text {th }}$ iteration of the rod position $\left(U^{(n)}\right)$ and the Lagrange multiplier $\left(\lambda^{(n)}\right)$.

$$
\left.\begin{array}{c}
\left(K_{i j l k}^{1}+\lambda_{n} K_{n i j l k}^{2}\right) U_{j k}^{(n)}-F_{i l}^{e}=0=G \\
A_{m i l} U_{j l}^{(n)} U_{j k}^{(n)}-B_{m}-E_{m i l}\left(U^{(n)}, 0\right)-C_{m n} \lambda_{n}^{(n)}=0=H
\end{array}\right\}
$$

To solve the equation, a Newton-Raphson's iterative method is used. Using Taylor series expansion, the equation system can be written using the first order term as:

$$
\left.\begin{array}{l}
G_{i l}^{(n+1)}=G_{i l}^{(n)}+\frac{\partial G_{i l}}{\partial U_{j k}} \Delta U_{j k}+\frac{\partial G_{i l}}{\partial \lambda_{n}} \Delta \lambda_{n}=0 \\
H_{m}^{(n+1)}=H_{m}^{(n)}+\frac{\partial H_{m}}{\partial U_{j k}} \Delta U_{j k}+\frac{\partial H_{m}}{\partial \lambda_{n}} \Delta \lambda_{n}=0
\end{array}\right\}
$$

Where:

$$
\begin{aligned}
& \frac{\partial G_{i l}}{\partial U_{j k}}=K_{i j l k}^{1}+\frac{\partial K_{i j l k}^{1}}{\partial U} U_{j k}^{(n)}+\lambda_{n}^{(n)} K_{n i j l k}^{2}+\lambda_{n}^{(n)} \frac{\partial K_{n i j l k}^{2}}{\partial U} U_{j k}^{(n)} \\
& \frac{\partial G_{i l}}{\partial \lambda_{n}}=K_{n i j l k}^{2} U_{j k}^{(n)} \\
& \frac{\partial H_{m}}{\partial U_{j k}}=2 A_{m k l} U_{j k}^{(n)}-\frac{\partial E_{m i l}\left(U_{j k}^{(n)}, 0\right)}{\partial U} \\
& \frac{\partial H_{m}}{\partial \lambda_{n}}=-C_{m n} \\
& \frac{\partial K_{i j l k}^{1}}{\partial U}=\sum_{i=1}^{n} \int_{0}^{L}-E I N_{l}^{\prime \prime} N_{k}^{\prime \prime} U_{j k}^{(n)} \frac{U_{j s}{ }^{T} N_{s}^{\prime T} \cdot N_{t}^{\prime}}{\left(\left(N_{s}^{\prime} U_{j s}\right)^{T} \cdot N_{t}^{\prime} U_{j t}\right)^{\frac{3}{2}}} \delta_{i j} d s
\end{aligned}
$$




$$
\begin{aligned}
& \frac{\partial K_{i j l k}^{2}}{\partial U} U_{j k}^{(n)}=\sum_{i=1}^{n} \int_{0}^{L}-P_{n} N_{l}^{\prime} N_{k}^{\prime} U_{j k}^{(n)} \frac{U_{j s}{ }^{T} N_{s}^{\prime T} \cdot N_{t}^{\prime}}{\left(\left(N_{s}^{\prime} U_{j s}\right)^{T} \cdot N_{t}^{\prime} U_{j t}\right)^{\frac{3}{2}}} \delta_{i j} d s \\
& \frac{\partial E_{m i l}(U, 0)}{\partial U}=\frac{E I}{E A} \sum_{i=1}^{n} \int_{0}^{L} P_{m}\left(\frac{2 \cdot\left(N_{s}^{\prime \prime} U_{j s}\right)^{T} \cdot N_{t}^{\prime \prime}}{\left(N_{s}^{\prime} U_{j s}\right)^{T} \cdot N_{t}^{\prime} U_{i t}}-\frac{\left(N_{s}^{\prime \prime} U_{j s}\right)^{T} \cdot N_{t}^{\prime \prime} U_{i t} \cdot 2\left(N_{s}^{\prime} U_{j s}\right)^{T} \cdot N_{t}^{\prime}}{\left(\left(N_{s}^{\prime} U_{j s}\right)^{T} \cdot N_{t}^{\prime} U_{i t}\right)^{2}}\right) d s
\end{aligned}
$$

Then, the system of Eq. (52) can be written in matrix form as:

$$
\left[\begin{array}{cc}
K_{i j l k}^{1}+\frac{\partial K_{i j l k}^{1}}{\partial U} U_{j k}^{(n)}+\lambda_{n} K_{n i j l k}^{2}+\lambda_{n} \frac{\partial K_{n i j l k}^{2}}{\partial U} U_{j k}^{(n)} & K_{n i j l k}^{2} U_{j k}^{(n)} \\
2 A_{m k l} U_{j k}^{(n)}-\frac{\partial E_{m i l}\left(U_{j k}^{(n)}, 0\right)}{\partial U} & -C_{m n}
\end{array}\right]\left[\begin{array}{c}
\Delta U_{j k} \\
\Delta \lambda
\end{array}\right]=\left[\begin{array}{c}
-G_{i l}^{(n)} \\
-H_{m}^{(n)}
\end{array}\right]
$$

And the next iterative solution is expressed as follows:

$$
\left.\begin{array}{c}
U_{j k}^{(n+1)}=U_{j k}^{(n)}+\Delta U_{j k} \\
\lambda_{n}^{(n+1)}=\lambda_{n}^{(n)}+\Delta \lambda_{n}
\end{array}\right\}
$$

Then, from an initial approximation of the rod position shape $\left(U^{0}\right)$ and the Lagrange multiplier $\left(\lambda^{0}\right)$ the static values for a given loading parameter are obtained.

\section{Time integration of dynamic equations}

The time integration scheme is based on an implicit generalised Newmark's method [37]. The method is applied in a non-linear context with a corrector procedure at each time-step using the Newton algorithm.

The method has to satisfy the dynamic equilibrium in each time step by approximating the position of the $(n+1)$ step $\left(U^{n+1}\right)$ from the previous time step $\left(U^{n}\right)$. The Newmark's time integration procedure involves the next equations:

$$
\begin{aligned}
& U_{n+1}=U_{n}+\Delta t \dot{U}_{n}+\frac{\Delta t}{2}\left((1-2 \beta) \ddot{U}_{n}+2 \beta \ddot{U}_{n+1}\right) \\
& \dot{U}_{n+1}=\dot{U}_{n}+\Delta t\left((1-\gamma) \ddot{U}_{n}+\gamma \ddot{U}_{n+1}\right)
\end{aligned}
$$

where $\beta$ and $\gamma$ are the Newmark constants, and $\Delta t$ is the incremental time step.

Then, the velocity and acceleration parameters of the next time step can be assessed as a function of the increment of the position $\Delta U=U_{n+1}-U_{n}$

$$
\left.\begin{array}{c}
\dot{U}_{n+1}=C_{v}+\frac{\gamma}{\beta \Delta t} \Delta U \\
\ddot{U}_{n+1}=C_{a}+\frac{1}{\beta \Delta t^{2}} \Delta U
\end{array}\right\}
$$

where $C_{v}$ and $C_{a}$ can be defined as:

$$
\begin{aligned}
C_{v} & =\dot{U}_{n}+(1-\gamma) \Delta t \ddot{U}_{n}-\gamma\left(\Delta t \dot{U}_{n}+\left(\frac{1}{2}-\beta\right) \Delta t^{2} \ddot{U}_{n}\right) /(\beta \Delta t) \\
C_{a} & =-\left(\Delta t \ddot{U}_{n}+\left(\frac{1}{2}-\beta\right) \Delta t^{2} \ddot{U}_{n}\right) /\left(\beta \Delta t^{2}\right)
\end{aligned}
$$

The dynamic equation can be expressed from Eq. $(40)$ as follows at the $(n+1)$ time step: 


$$
\begin{aligned}
& M_{i j l k} \ddot{U}_{j k}^{(n+1)}+F_{i l}^{i(n+1)}-F_{i l}^{e(n+1)}=G_{i l}^{(n+1)}=0 \\
& A_{m i l} U_{k l}^{(n+1)} U_{k i}^{(n+1)}-B_{m}+D_{m n} U_{k l}^{(n+1)} \dot{U}_{k i}^{(n+1)}-E_{m i l}\left(U^{(n+1)} \dot{U}^{(n+1)}\right)- \\
& C_{m n} \lambda_{n}^{(n+1)} H_{m}^{(n+1)}=0
\end{aligned}
$$

Where $F_{i l}^{i(n+1)}$ are the internal forces, $F_{i l}^{e(n+1)}$ are the external forces and $G_{i l}^{(n+1)}$ are the equivalent dynamic out-of-balance forces at the $(n+1)$ time step.

The internal forces and the constitutive equation can be approximate using truncated Taylor series as shown in Eq. (67) and Eq. (68).

$$
\begin{aligned}
& F_{i l}^{i(n+1)}=F_{i l}^{i(n)}+\frac{\partial F_{i l}}{\partial U_{j k}} \Delta U_{j k}+\frac{\partial F_{i l}}{\partial \lambda_{n}} \Delta \lambda_{n}=F_{i l}^{i(n)}+\left(K_{i j l k}^{1}+\frac{\partial K_{i j l k}^{1}}{\partial U} U_{j k}^{(n)}+K_{i j l k}^{1, D a m p}+\right. \\
& \left.\frac{\partial K_{i j l k}^{1, D a m p}}{\partial U} U_{j k}^{(n)}+\lambda_{n}^{(n)} \frac{\partial K_{n i j l k}^{2}}{\partial U} U_{j k}^{(n)}+\lambda_{n}^{(n)} K_{n i j l k}^{2}\right) \Delta U_{j k}+K_{n i j l k}^{2} U_{j k}^{(n)} \cdot \Delta \lambda_{n} \\
& H_{m}^{(n+1)}=H_{m}^{(n)}+\frac{\partial H_{m}}{\partial U_{j k}} \Delta U_{j k}+\frac{\partial H_{m}}{\partial \lambda_{n}} \Delta \lambda_{n}=H_{m}^{(n)}+\left(2 A_{m i l} U_{k l}^{(n)}+D_{m n}\left(\dot{U}_{k i}^{(n)}+\frac{\gamma}{\beta \Delta t} U_{k l}^{(n)}\right)-\right. \\
& \left.\frac{\partial E_{m i l}}{\partial U}\right) \cdot \Delta U_{j k}-C_{m n} \Delta \lambda_{n}
\end{aligned}
$$

where:

$$
\begin{aligned}
& \frac{\partial K_{i j l k}^{1}}{\partial U} U_{j k}^{(n)}=\int_{0}^{L}-E I N_{l}^{\prime \prime} N_{k}^{\prime \prime} U_{j k}^{(n)} \frac{U_{j s}{ }^{T} N_{s}^{\prime T} \cdot N_{t}^{\prime}}{\left(\left(N_{s}^{\prime} U_{j s}\right)^{T} \cdot N_{t}^{\prime} U_{j t}\right)^{\frac{3}{2}}} \delta_{i j} d s \\
& \frac{\partial K_{i j l k}^{1, \text { Damp }}}{\partial U} U_{j k}^{(n)}=\int_{0}^{L} \eta_{B} I \frac{N_{l}^{\prime \prime} N_{k}^{\prime \prime}}{\sqrt{\left(N_{s}^{\prime} U_{j s}\right)^{T} \cdot N_{t}^{\prime} U_{i t}}} U_{j k}^{(n)}\left(\frac{\left(N_{s}^{\prime \prime} \dot{U}_{i t}\right)^{T} \cdot N_{t}^{\prime \prime}+\frac{\gamma}{\beta \Delta t}\left(N_{s}^{\prime \prime} U_{i t}\right)^{T} \cdot N_{t}^{\prime \prime}}{\left(N_{s}^{\prime \prime} U_{j s}\right)^{T} \cdot N_{t}^{\prime \prime} U_{i t}}-\right. \\
& \left.\frac{\left(N_{s}^{\prime \prime} U_{j s}\right)^{T} \cdot N_{t_{i t}^{\prime \prime}} \cdot 2\left(N_{s}^{\prime \prime} U_{i t}\right)^{T} \cdot N_{t}^{\prime \prime}}{\left(\left(N_{s}^{\prime \prime} U_{j s}\right)^{T} \cdot N_{t}^{\prime \prime} U_{i t}\right)^{2}}-\left(\frac{\left(N_{s}^{\prime} \dot{U}_{i t}\right)^{T} \cdot N_{t}^{\prime}+\frac{\gamma}{\beta \Delta t}\left(N_{s}^{\prime} U_{i t}\right)^{T} \cdot N_{t}^{\prime}}{\left(N_{s}^{\prime} U_{j s}\right)^{T} \cdot N_{t}^{\prime} U_{i t}}-\frac{\left(N_{s}^{\prime} U_{j s}\right)^{T} \cdot N_{t}^{\prime} \dot{U}_{i t} \cdot 2\left(N_{s}^{\prime} U_{j s}\right)^{T} \cdot N_{t}^{\prime}}{\left(\left(N_{s}^{\prime} U_{j s}\right)^{T} \cdot N_{t}^{\prime} U_{i t}\right)^{2}}\right)\right)- \\
& \eta_{B} I N_{l}^{\prime \prime} N_{k}^{\prime \prime} U_{j k}^{(n)}\left(\frac{\left(N_{s}^{\prime \prime} U_{j s}\right)^{T} \cdot N_{t i t}^{\prime \prime}}{\left(N_{s}^{\prime \prime} U_{j s}\right)^{T} \cdot N_{t}^{\prime \prime} U_{i t}}-\frac{\left(N_{s}^{\prime} U_{j s}\right)^{T} \cdot N_{t}^{\prime} \dot{U}_{i t}}{\left(N_{s}^{\prime} U_{j s}\right)^{T} \cdot N_{t}^{\prime} U_{i t}}\right) \frac{U_{j s}{ }^{T} N_{s}^{\prime}{ }^{T} \cdot N_{t}^{\prime}}{\left(\left(N_{s}^{\prime} U_{j s}\right)^{T} \cdot N_{t}^{\prime} U_{j t}\right)^{\frac{3}{2}}} \delta_{i j} d s \\
& \frac{\partial K_{i j l k}^{2}}{\partial U} U_{j k}^{(n)}=\int_{0}^{L}-P_{n} N_{l}^{\prime} N_{k}^{\prime} U_{j k}^{(n)} \frac{U_{j s}{ }^{T} N_{s}^{\prime T} \cdot N_{t}^{\prime}}{\left(\left(N_{s}^{\prime} U_{j s}\right)^{T} \cdot N_{t}^{\prime} U_{j t}\right)^{\frac{3}{2}}} \delta_{i j} d s \\
& \frac{\partial E_{m i l}}{\partial U}=\frac{E I}{E A} \int_{0}^{L} P_{m}\left(\frac{2 \cdot\left(N_{s}^{\prime \prime} U_{j s}\right)^{T} \cdot N_{t}^{\prime \prime}}{\left(N_{s}^{\prime} U_{j s}\right)^{T} \cdot N_{t}^{\prime} U_{i t}}-\frac{\left(N_{s}^{\prime \prime} U_{j s}\right)^{T} \cdot N_{t}^{\prime \prime} U_{i t} \cdot 2\left(N_{s}^{\prime} U_{j s}\right)^{T} \cdot N_{t}^{\prime}}{\left(\left(N_{s}^{\prime} U_{j s}\right)^{T} \cdot N_{t}^{\prime} U_{i t}\right)^{2}}\right) d s+ \\
& \frac{\eta_{B} I}{E A} \int_{0}^{L} P_{m}\left(\frac{\left(N_{s}^{\prime \prime} \dot{U}_{i t}\right)^{T} \cdot N_{t}^{\prime \prime}+\frac{\gamma}{\beta \Delta t}\left(N_{s}^{\prime \prime} U_{i t}\right)^{T} \cdot N_{t}^{\prime \prime}}{\left(N_{s}^{\prime \prime} U_{j s}\right)^{T} \cdot N_{t}^{\prime \prime} U_{i t}}-\frac{\left(N_{s}^{\prime \prime} U_{j s}\right)^{T} \cdot N_{t}^{\prime \prime} \dot{U}_{i t} \cdot 2\left(N_{s}^{\prime \prime} \dot{U}_{i t}\right)^{T} \cdot N_{t}^{\prime \prime}}{\left(\left(N_{s}^{\prime \prime} U_{j s}\right)^{T} \cdot N_{t}^{\prime \prime} U_{i t}\right)^{2}}-\left(\frac{\left(N_{s}^{\prime} \dot{U}_{i t}\right)^{T} \cdot N_{t}^{\prime}+\frac{\gamma}{\beta \Delta t}\left(N_{s}^{\prime} U_{i t}\right)^{T} \cdot N_{t}^{\prime}}{\left(N_{s}^{\prime} U_{j s}\right)^{T} \cdot N_{t}^{\prime} U_{i t}}-\right.\right. \\
& \left.\left.\frac{\left(N_{s}^{\prime} U_{j s}\right)^{T} \cdot N_{t}^{\prime} \dot{U}_{i t} \cdot 2\left(N_{s}^{\prime} U_{j s}\right)^{T} \cdot N_{t}^{\prime}}{\left(\left(N_{s}^{\prime} U_{j s}\right)^{T} \cdot N_{t}^{\prime} U_{i t}\right)^{2}}\right)\right) \frac{\left(N_{s}^{\prime \prime} U_{j s}\right)^{T} \cdot N_{t}^{\prime \prime} U_{i t}}{\left(N_{s}^{\prime} U_{j s}\right)^{T} \cdot N_{t}^{\prime} U_{i t}}+P_{m}\left(\frac{\left(N_{s}^{\prime \prime} U_{j s}\right)^{T} \cdot N_{t}^{\prime} \dot{U}_{i t}}{\left(N_{s}^{\prime \prime} U_{j s}\right)^{T} \cdot N_{t}^{\prime \prime} U_{i t}}-\frac{\left(N_{s}^{\prime} U_{j s}\right)^{T} \cdot N_{t}^{\prime} \dot{U}_{i t}}{\left(N_{s}^{\prime} U_{j s}\right)^{T} \cdot N_{t}^{\prime} U_{i t}}\right)\left(\frac{2 \cdot\left(N_{s}^{\prime \prime} U_{j s}\right)^{T} \cdot N_{t}^{\prime \prime}}{\left(N_{s}^{\prime} U_{j s}\right)^{T} \cdot N_{t}^{\prime} U_{i t}}-\right. \\
& \left.\frac{\left(N_{s}^{\prime \prime} U_{j s}\right)^{T} \cdot N_{t}^{\prime \prime} U_{i t} \cdot 2\left(N_{s}^{\prime} U_{j s}\right)^{T} \cdot N_{t}^{\prime}}{\left(\left(N_{s}^{\prime} U_{j s}\right)^{T} \cdot N_{t}^{\prime} U_{i t}\right)^{2}}\right) d s
\end{aligned}
$$

Then, substituting Eq. (64), Eq. (67), Eq. (68) into Eq. (66) we obtain: 


$$
\begin{aligned}
& M_{i j l k}\left(\frac{1}{\beta \Delta t^{2}} \Delta U+C_{a}\right)+F_{i l}^{i(n)}+\left(K_{i j l k}^{1}+\frac{\partial K_{i j l k}^{1}}{\partial U} U_{j k}^{(n)}+K_{i j l k}^{1, D a m p}+\frac{\partial K_{i j l k}^{1, D a m p}}{\partial U} U_{j k}^{(n)}+\right. \\
& \left.\lambda_{n}^{(n)} \frac{\partial K_{n i j l k}^{2}}{\partial U} U_{j k}^{(n)}+\lambda_{n}^{(n)} K_{n i j l k}^{2}\right) \Delta U_{j k}+K_{n i j l k}^{2} U_{j k}^{(n)} \cdot \Delta \lambda_{n}-F_{i l}^{e(n+1)}=0 \\
& \left(2 A_{m i l} U_{k l}^{(n)}+D_{m n}\left(\dot{U}_{k i}^{(n)}+\frac{\gamma}{\beta \Delta t} U_{k l}^{(n)}\right)-\frac{\partial E_{m i l}}{\partial U}\right) \Delta U_{j k}-C_{m n} \Delta \lambda_{n}=-H_{m}^{(n)}
\end{aligned}
$$

Rearranging the terms of Eq. (73)

$$
\begin{aligned}
& M_{i j l k} \frac{1}{\beta \Delta t^{2}} \Delta U+\left(K_{i j l k}^{1}+\frac{\partial K_{i j l k}^{1}}{\partial U} U_{j k}^{(n)}+K_{i j l k}^{1, D a m p}+\frac{\partial K_{i j l k}^{1, D a m p}}{\partial U} U_{j k}^{(n)}+\lambda_{n}^{(n)} \frac{\partial K_{n i j l k}^{2}}{\partial U} U_{j k}^{(n)}+\right. \\
& \left.\lambda_{n}^{(n)} K_{n i j l k}^{2}\right) \cdot \Delta U_{j k}+K_{n i j l k}^{2} U_{j k}^{(n)} \Delta \lambda_{n}=-M_{i j l k} C_{a}-F_{i l}^{i(n)}+F_{i l}^{e(n+1)} \\
& \left(2 A_{m i l} U_{k l}^{(n)}+D_{m n}\left(\dot{U}_{k i}^{(n)}+\frac{\gamma}{\beta \Delta t} U_{k l}^{(n)}\right)-\frac{\partial E_{m i l}}{\partial U}\right) \Delta U_{j k}-C_{m n} \Delta \lambda_{n}=-H_{m}^{(n)}
\end{aligned}
$$

The above equation can be rewritten in a matrix form as follows:

$$
K_{d y n} \Delta y=\Delta F_{d y n}
$$

where:

$$
\begin{aligned}
& K_{d y n}=\left[\begin{array}{cc}
M_{i j l k} \frac{1}{\beta \Delta t^{2}}+K_{i j l k}^{1}+\frac{\partial K_{i j l k}^{1}}{\partial U} U_{j k}^{(n)}+K_{i j l k}^{1, D a m p}+\frac{\partial K_{i j l k}^{1, D a m p}}{\partial U} U_{j k}^{(n)}+\cdots & K_{n i j l k}^{2} U_{j k}^{(n)} \\
\cdots+\lambda_{n}^{(n)} \frac{\partial K_{n i j l k}^{2}}{\partial U} U_{j k}^{(n)}+\lambda_{n}^{(n)} K_{n i j l k}^{2} & \\
2 A_{m i l} U_{k l}^{(n)}+D_{m n}\left(\dot{U}_{k i}^{(n)}+\frac{\gamma}{\beta \Delta t} U_{k l}^{(n)}\right)-\frac{\partial E_{m i l}}{\partial U} & -C_{m n}
\end{array}\right] \\
& \Delta F_{d y n}=\left[\begin{array}{c}
-M_{i j l k} C_{a}-\left(K_{i j l k}^{1}+K_{i j l k}^{1, D a m p}+\lambda_{n} K_{n i j l k}^{2}\right) U_{j k}^{(n)}+F_{i l}^{e(n+1)} \\
-H_{m}^{(n)}
\end{array}\right] \\
& \Delta y=\left[\begin{array}{c}
\Delta U_{j k} \\
\Delta \lambda_{n}
\end{array}\right]
\end{aligned}
$$

Then, a first approach of the $y^{n+1}$ can be assessed as $y^{n+1}=y^{n}+\Delta y$.

To ensure the convergence of the $(n+1)$ time step, a corrector procedure is implemented from the unbalanced residual of Eq. (66) by a Newton-Rapson corrector. Suppose to be $j$, the $j-t h$ iteration of corrector procedure, Eq. (66) can be expressed as:

$$
\begin{aligned}
& M_{i j l k} \ddot{U}_{j k}^{(n+1, j)}+F_{i l}^{i(n+1, j)}-F_{i l}^{e(n+1, j)}=G_{i l}^{(n+1, j)}=0 \\
& A_{m i l} U_{k l}^{(n+1, j)} U_{k i}^{(n+1, j)}-B_{m}+D_{m n} U_{k l}^{(n+1, j)} \dot{U}_{k i}^{(n+1, j)}-E_{m i l}\left(U^{(n+1, j)} \dot{U}^{(n+1, j)}\right)- \\
& C_{m n} \lambda_{n}^{(n+1, j)}=H_{m}^{(n+1, j)}=0
\end{aligned}
$$

Then, the $(j+1)$ unbalanced term of the $(n+1)$ time step can be defined as Eq. (81)

$$
\begin{aligned}
& {\left[\begin{array}{l}
G_{i l}^{(n+1, j+1)} \\
H_{m}^{(n+1, j+1)}
\end{array}\right]=\left[\begin{array}{l}
G_{i l}^{(n+1, j)} \\
H_{m}^{(n+1, j)}
\end{array}\right]+K_{d y n} \Delta y^{j+1}=\left[\begin{array}{l}
0 \\
0
\end{array}\right]} \\
& \Delta y^{j+1}=K_{d y n}^{-1}\left[\begin{array}{l}
-G_{i l}^{(n+1, j)} \\
-H_{m}^{(n+1, j)}
\end{array}\right]
\end{aligned}
$$


And the $j+1-t h$ approximation can be assessed as next:

$$
\begin{aligned}
& y^{(n+1, j+1)}=y^{(n+1, j)}+\Delta y^{j+1} \\
& \dot{y}^{(n+1, j+1)}=\dot{y}^{(n+1, j)}+\frac{\gamma}{\beta \Delta t} \Delta y^{j+1} \\
& \ddot{y}^{(n+1, j+1)}=\ddot{y}^{(n+1, j)}+\frac{1}{\beta \Delta t^{2}} \Delta y^{j+1}
\end{aligned}
$$

Where at every $(j+1)-t h$ iteration the stiffness matrices, and forces are updated.

The FEM implementation presented above allows to take into account the fully dynamics of the rod system adding the rheological damping of both Axial and Bending strengths.

\section{Numerical verification}

The numerical verification of the model is based on two plus one examples. The first two cases mobilise the axial damping and the bending damping of the same bar, and the third case mobilises both, axial and bending damping trough a post buckling state. All the simulations are performed for a range form 0 to $100 \%$ of the critical damping.

\subsection{Axial and Bending Damping}

The axial and bending damping verification test are a numerical simulation performed by applying an initial axial strain and bending deformation to a bar, which is allowed to move freely. The properties of the bar are shown in Table 1, and is discretized in 20 elements.

\subsubsection{Axial case}

The axial case is performed by imposing an initial strain equivalent to a $500 \mathrm{~N}$ tension at the free end as shown in Figure 3. Six different axial damping viscosity ratios are used to compare the axial damping behaviour. According to section 3.3, the axial critical viscosity for a cantilever beam can be computed as $\eta_{A, \text { Critic }}=\frac{4 L}{\pi} \sqrt{\rho_{m} \cdot E}$ which takes a value of $\eta_{A, \text { Critic }}=2.576 \cdot 10^{7}\left[\mathrm{~N} /\left(\mathrm{m}^{2} \mathrm{~s}\right)\right]$.

The time step used in the simulation is $0.5 \cdot 10^{-5} s$ (approximately one hundredth of the un-damped axial natural period of the bar) and the convergence of the dynamics of the rod was achieved after an average of two iterations. 


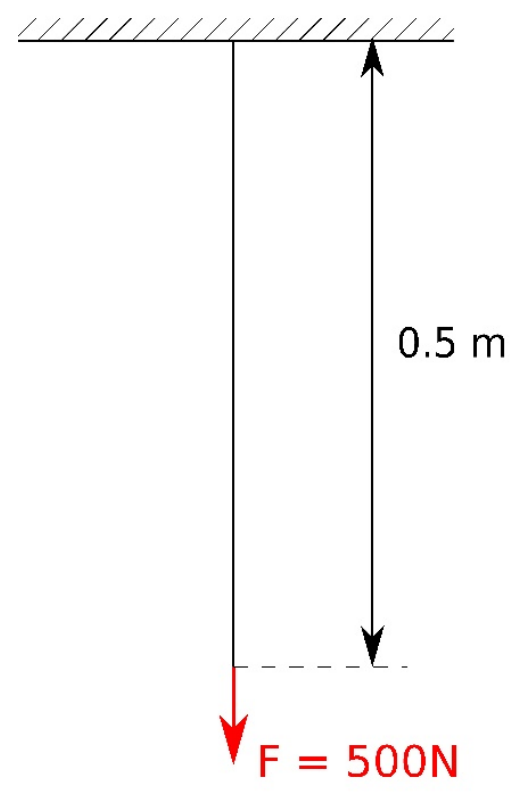

\begin{tabular}{|l|l|}
\hline Parameter & Value \\
\hline Length $[\mathrm{m}]$ & 0.5 \\
\hline Diameter $[\mathrm{m}]$ & 0.02 \\
\hline $\mathrm{A}\left[\mathrm{m}^{2}\right]$ & $3.14 \mathrm{E}-04$ \\
\hline $\mathrm{I}\left[\mathrm{m}^{4}\right]$ & $7.85 \mathrm{E}-09$ \\
\hline$\rho\left[\mathrm{kg} / \mathrm{m}^{3}\right]$ & 7800.00 \\
\hline $\mathrm{E}\left[\mathrm{N} / \mathrm{m}^{2}\right]$ & $2.10 \mathrm{E}+11$ \\
\hline Mass per meter length $[\mathrm{kg} / \mathrm{m}]$ & 2.46 \\
\hline Total mass $[\mathrm{kg}]$ & 1.23 \\
\hline EA $[\mathrm{N}]$ & $6.60 \mathrm{E}+07$ \\
\hline EI $\left[\mathrm{N} \cdot \mathrm{m}^{2}\right]$ & $1.65 \mathrm{E}+03$ \\
\hline
\end{tabular}

Table 1: Parameters of the rod for the axial and bending verification test

Figure 3: Sketch of the axial verification test

The initial linear elastic displacement at the free end is $\Delta z=L \cdot \frac{F}{E A}=3.79 \cdot 10^{-3} \mathrm{~mm}$. Figure 4 shows the results of the elongation at the free in the time domain, with the different ration of damping. From $0 \%$ of the axial critical damping to a $100 \%$ ratio, the elongation of the free end varies from a sinusoidal behaviour to an overdamped motion. For the intermediate damping ratios, the more axial damping ratio the more amplitude is reduced. Moreover, the natural period of the un-damped motion of the simulation can be assessed from Eq. (23) by $T=\frac{2 \pi}{\omega_{n}}=\frac{2 \pi}{\sqrt{k / m}}=\frac{2 \pi}{\sqrt{E \beta_{A}^{2} / \rho}}=0.000385 \mathrm{~s}=0.385 \mathrm{~ms}$, where $\beta_{A}=\frac{\pi}{2 L}$, which shows good agreement with the simulations. 


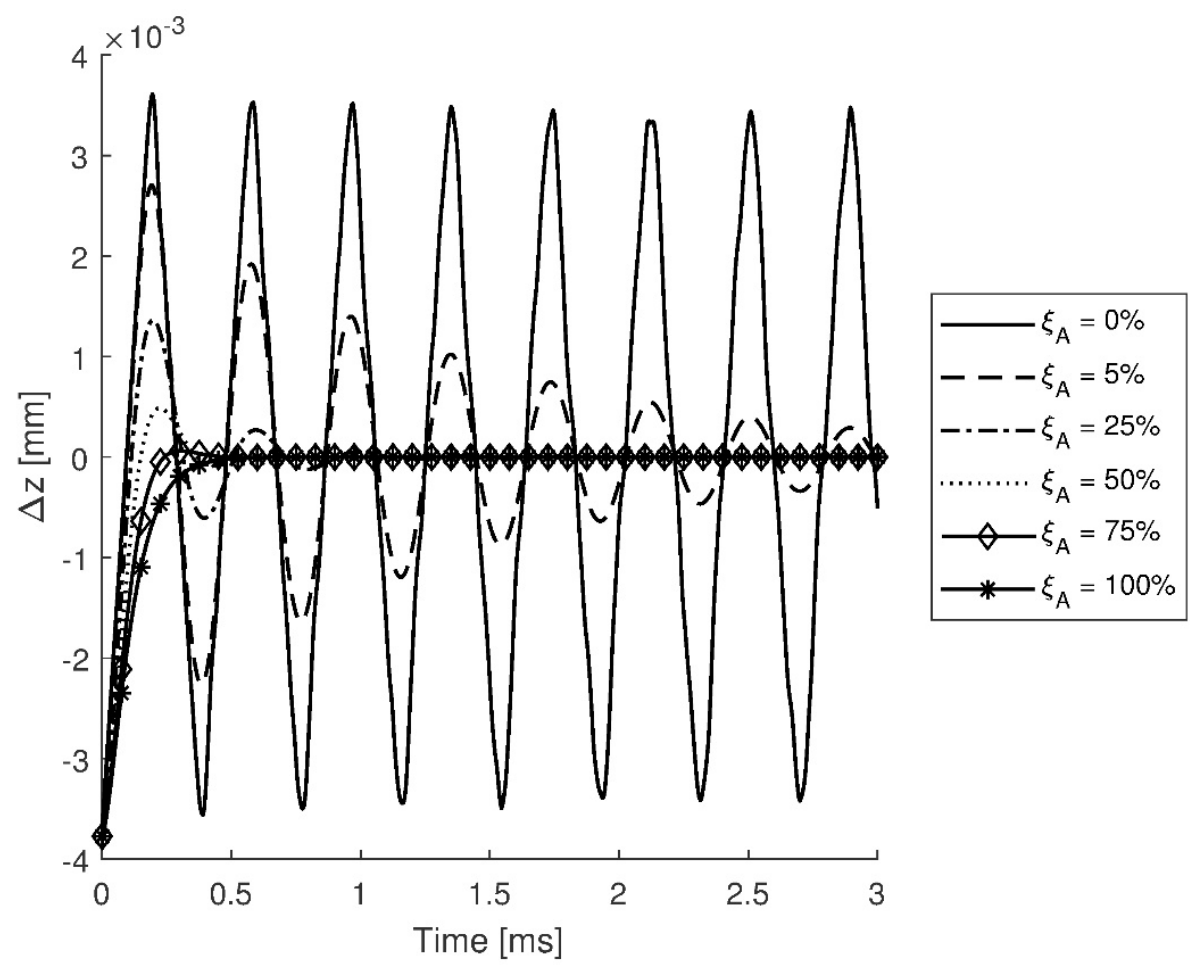

Figure 4: Incremental position of the free-end node of the rod

\subsubsection{Bending case}

The bending case is performed by imposing a bending moment at the free end of $\mathrm{M}=172.8 \mathrm{~N} \cdot \mathrm{m}$ as is shown in Figure 5. According to section 3.3, the bending critical viscosity for a cantilever beam can be computed as $\eta_{B, \text { Critic }}=2 / \beta_{\mathrm{B}}^{2} \sqrt{\rho_{m} \cdot A E / I}$ which takes a value of $\eta_{\beta, \text { Critic }}=2.519 \cdot 10^{9}\left[\mathrm{~N} /\left(\mathrm{m}^{2} \mathrm{~s}\right)\right]$ for a $\beta_{B, 1}=0.59686 \pi / L$.

The time step used in the simulation is $1 \cdot 10^{-4} s$ (approximately one hundredth of the natural period of the $1^{\text {st }}$ bending mode) and the convergence of the dynamics of the rod was achieved after an average of three iterations.

The Figure 6 shows the $X$ position of the free end for the different damping ratios. For a $100 \%$ damping ratio, the system is overdamped while for a $0 \%$ damping ratio the motion is clearly un-damped.

Moreover, the $0 \%$ damping ratio does not present a clearly sinusoidal motion as the axial damping does. The reason is that the bending motion can be composed by more than one mode, which are more likely to appear for un-damped systems.

In order to compare the natural frequencies of each node with the theoretical ones, a Fast Fourier Transform (FFT) is applied to the $X$ motion of the free end node. Figure 7 shows the amplitudefrequency relation of the FFT and the main values of the peaks. The comparison of the theoretical frequency values with the values obtained through the FFT, in Table 2, presents a good match for all the cases and confirms the origin of the fluctuation of the motion of the free end. 
The theoretical natural frequencies of the un-damped motion of the bending modes of a cantilever beam can be assessed from Eq. (23) by $f=\frac{\omega_{n}}{2 \pi}=\frac{\sqrt{k / m}}{2 \pi}=\frac{\sqrt{E I \beta_{B}^{4} /(\rho A)}}{2 \pi}$

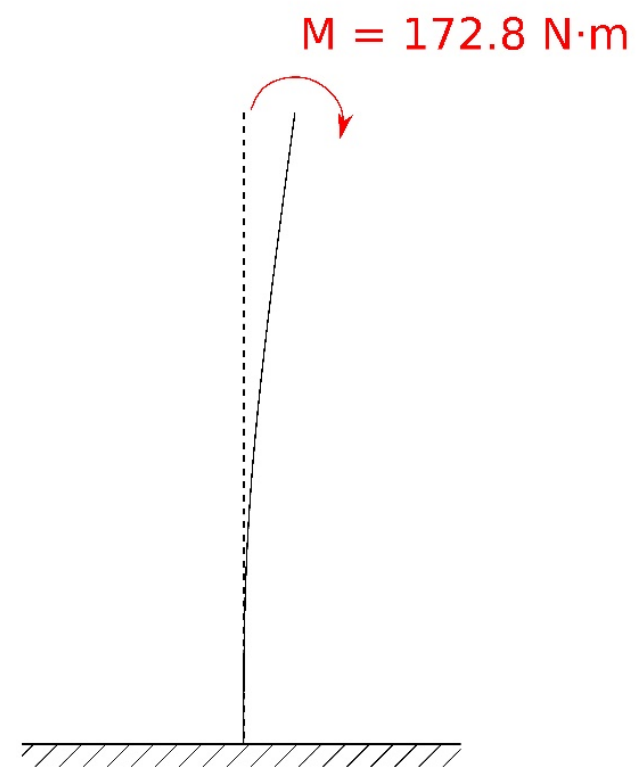

Figure 5: Sketch of the Bending verification test

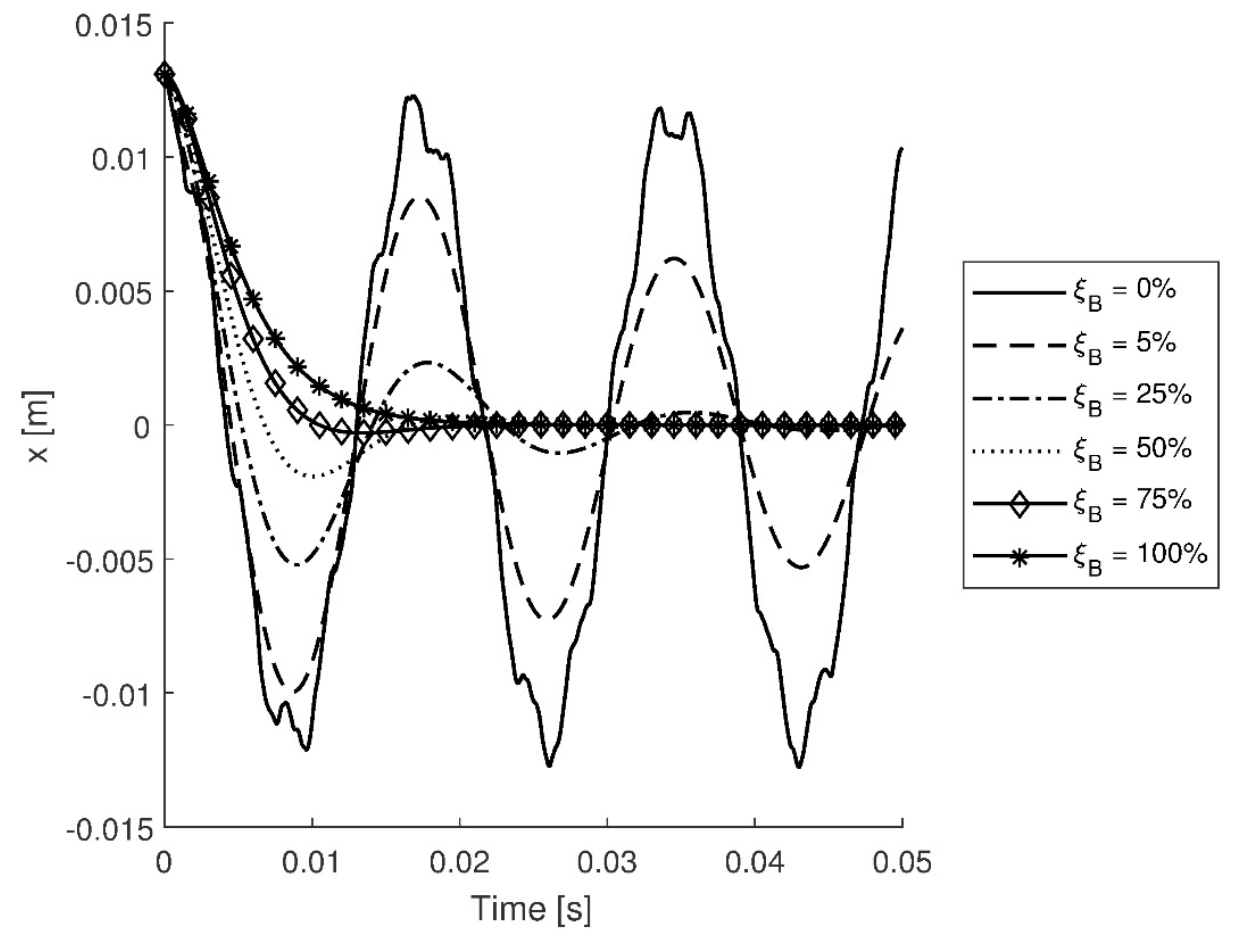

Figure 6: $X$ position of the free end node 


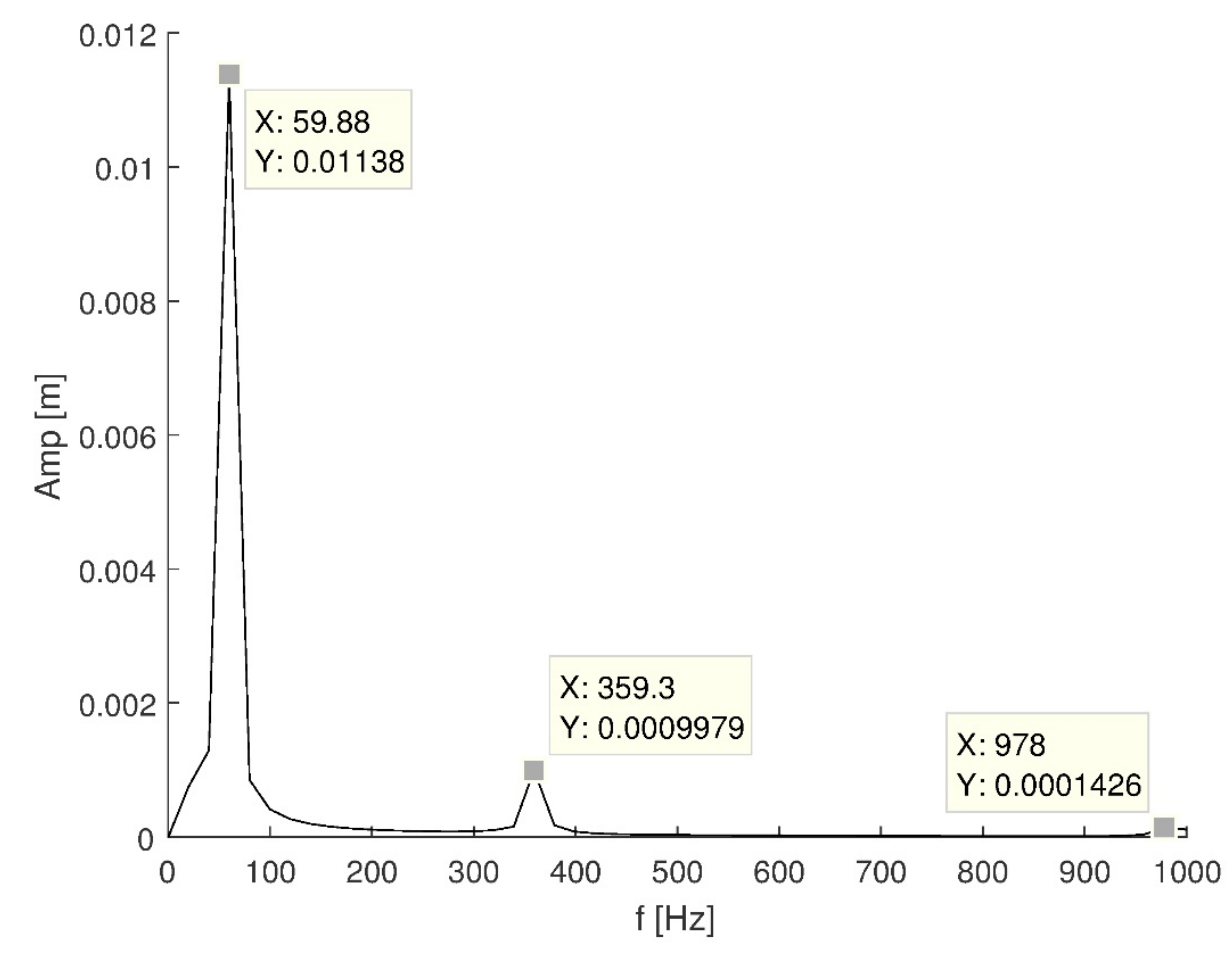

Figure 7: FFT of X motion of the free end node

\begin{tabular}{|l|c|c|c|}
\hline Mode\Parameter & $\boldsymbol{\beta}_{\boldsymbol{n}}$ & $\boldsymbol{f}_{\boldsymbol{n}}[\mathrm{Hz}]$ (theoretical) & $\boldsymbol{f}_{\boldsymbol{n}}[\mathrm{Hz}](\mathrm{FFT})$ \\
\hline $1^{\text {st }}$ mode $(\mathrm{n}=1)$ & 3.7502 & 57.97 & 59.88 \\
\hline $2^{\text {nd }}$ mode $(\mathrm{n}=2)$ & 9.3882 & 363.29 & 359.3 \\
\hline $3^{\text {rd }}$ mode $(\mathrm{n}=3)$ & 15.7095 & 1017 & 978 \\
\hline
\end{tabular}

Table 2: natural frequency mode comparison

\subsection{Post buckling behaviour - Axial and Bending coupled damping}

The coupled axial and bending damping verification test is a numerical simulation performed by imposing an initial deformation of a column equivalent to a post buckling state and allowing it to move freely. The initial deformation is achieved by applying an initial axial load higher than the critical buckling load as is shown in Figure 8. This state causes a linear decreasing bending moment through the column with the horizontal position, which is maximum on the base and 0 at the free end. Moreover, such as an initial axial load is applied, the axial damping is also mobilised. The properties of the column and its critical buckling axial force $\left(P_{c}\right)$ are shown in Table 3. The axial force applied is 1.0154 times the critical buckling force, that can be assessed by Eq. (83) [38].

The critical bending damping is assessed by Eq. (33) with the boundary conditions of a clumped end and a free end, which leads to a $\beta_{B}=0.59686 \pi / L$. Herein the viscosity term for the axial damping used is the one obtained through the bending critical damping as is the main load affected. Then, the critical viscosity used takes a value of $\eta_{\beta, \text { Critic }}=1.95 \cdot 10^{6}\left[\mathrm{~N} /\left(\mathrm{m}^{2} \mathrm{~s}\right)\right]$

$$
P_{\text {crit }}=\frac{\pi^{2} E I}{4 l^{2}}
$$


The simulation was performed with 20 elements for the bar discretization, the time step used in the simulation is $0.01 \mathrm{~s}$ and the convergence of the dynamics of the rod was achieved after an average of 4 iterations

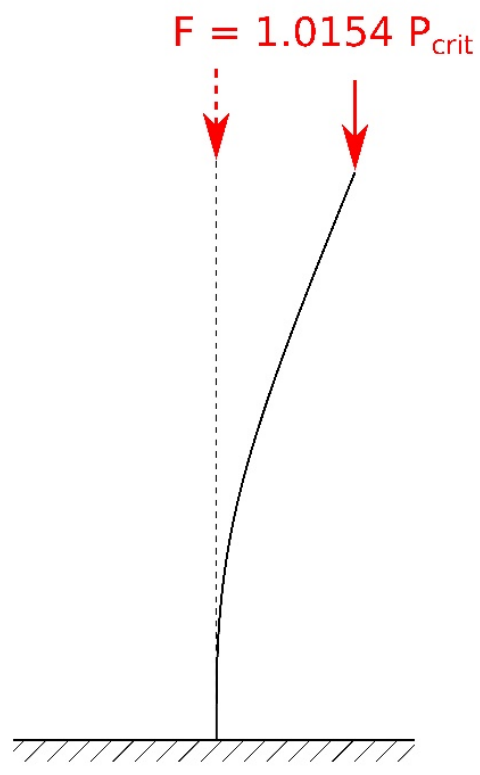

Figure 8: Sketch of the buckling verification test

\begin{tabular}{|l|l|}
\hline Parameter & Value \\
\hline Length $[\mathrm{m}]$ & 1 \\
\hline Diameter $[\mathrm{m}]$ & 0.08 \\
\hline $\mathrm{A}\left[\mathrm{m}^{2}\right]$ & $5.03 \mathrm{E}-03$ \\
\hline $\mathrm{I}\left[\mathrm{m}^{4}\right]$ & $2.01 \mathrm{E}-06$ \\
\hline$\rho\left[\mathrm{kg} / \mathrm{m}^{3}\right]$ & 1430 \\
\hline $\mathrm{E}\left[\mathrm{N} / \mathrm{m}^{2}\right]$ & $3.14 \mathrm{E}+06$ \\
\hline Mass per meter length $[\mathrm{kg} / \mathrm{m}]$ & 7.19 \\
\hline Total mass $[\mathrm{kg}]$ & 7.19 \\
\hline EA $[\mathrm{N}]$ & $1.54 \mathrm{E}+04$ \\
\hline El $\left[\mathrm{N} \cdot \mathrm{m}^{2}\right]$ & 6.021 \\
\hline$\eta_{B, C r i t i c}\left[\mathrm{~N} /\left(\mathrm{m}^{2} \mathrm{~s}\right)\right]$ & $1.951 \mathrm{e}+06$ \\
\hline$\xi_{B}($ damping ratio $)[\%]$ & $0-100$ \\
\hline $\mathrm{P}_{\text {crit }}[\mathrm{N}]$ & 14.86 \\
\hline $\mathrm{F}[\mathrm{N}]$ & 15.1 \\
\hline
\end{tabular}

Table 3: Parameters of the buckling verification test

The equilibrium point of the post buckling state for the initial axial force match the theoretical point [14], shown in Table 4. Figure 9 and Figure 10 show the horizontal and vertical position of the free end node during the simulation, which allow to compare the motions of the free end for different bending damping ratios. By and large, the more damping ratio the more free end motion is restrained. However, it is clearly state that a $100 \%$ bending damping ratio does not produces an overdamped behaviour of the column motion. This behaviour can be explained by the differences between the simulation test and the differential equation used for the derivation of the critical bending damping. In the simulation the column is not purely bending loaded, but axially loaded that produces a buckling state and thus an internal bending moment. Then, when the free end is released the column does not behave as if was loaded in pure bending. Moreover, for the $0 \%$ bending damping ratio, this phenomenon is accentuated due to the lack of damping. In both Figure 9 and Figure 10 is stated that the free end does not fit a perfect sinusoidal diagram. For this case, the deformed shape of the column in an instant, presents different curvature directions due to the superposition of different mode shapes as is shown in Figure 11.

The natural period of the un-damped motion of the bending simulation can be assessed from equation (23) by $T=\frac{2 \pi}{\omega_{n}}=\frac{2 \pi}{\sqrt{k / m}}=\frac{2 \pi}{\sqrt{E I \beta_{B}^{4} /(\rho A)}}=1.953 \mathrm{~s}$. 


\begin{tabular}{|l|l|l|l|}
\hline Model & \multicolumn{1}{|c|}{$\boldsymbol{P} / \boldsymbol{P}_{\text {crit }}$} & \multicolumn{1}{|c|}{$\boldsymbol{Z} / \boldsymbol{l}$} & \multicolumn{1}{|l|}{$/ \boldsymbol{l}$} \\
\hline Garret & 1.015397 & 0.9697 & 0.2194 \\
\hline Proposed Model & 1.015397 & 0.9690 & 0.2173 \\
\hline
\end{tabular}

Table 4: Position of free end node for a load above the critical buckling

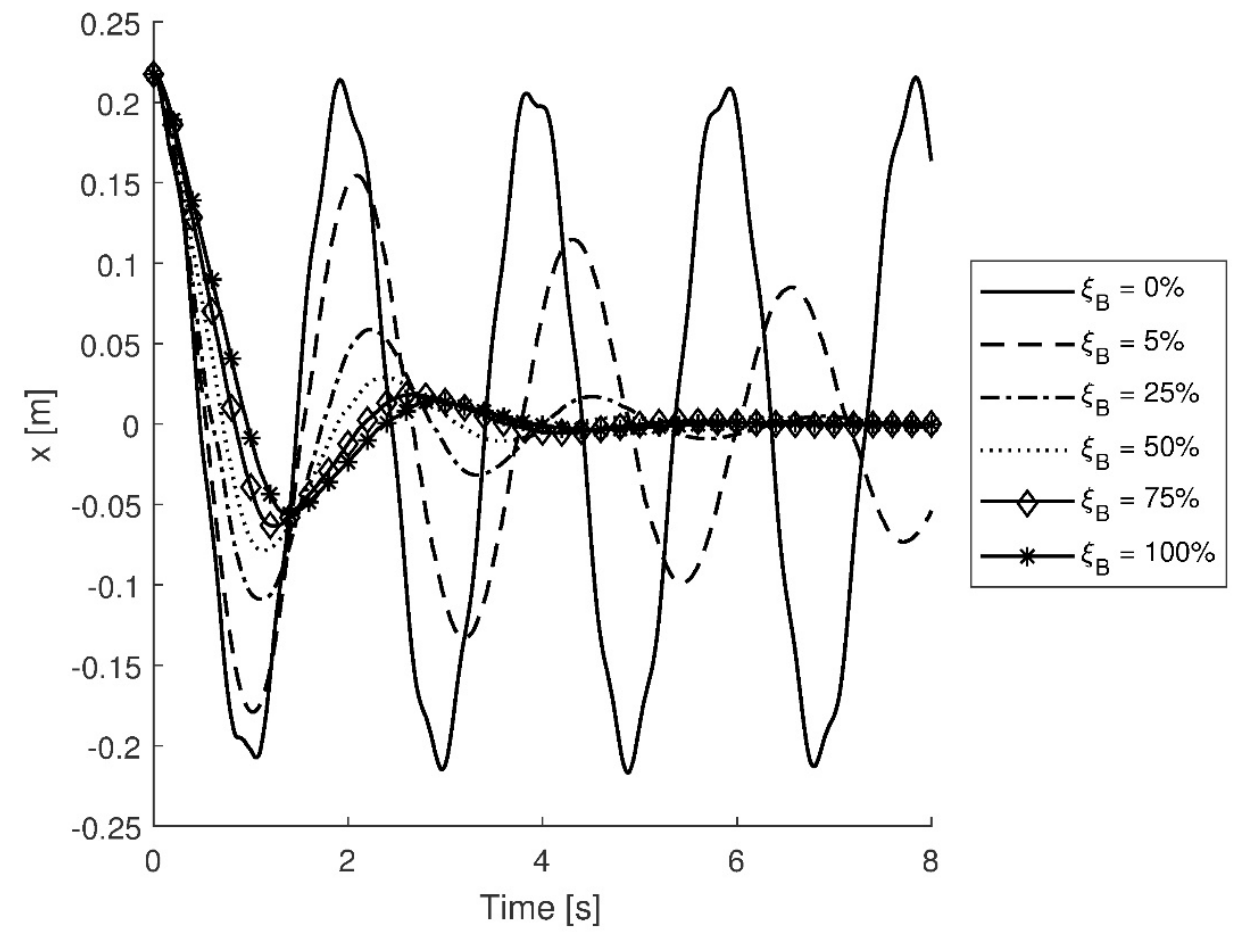

Figure 9: $X$ position of the free end along the time for different damping ratios 


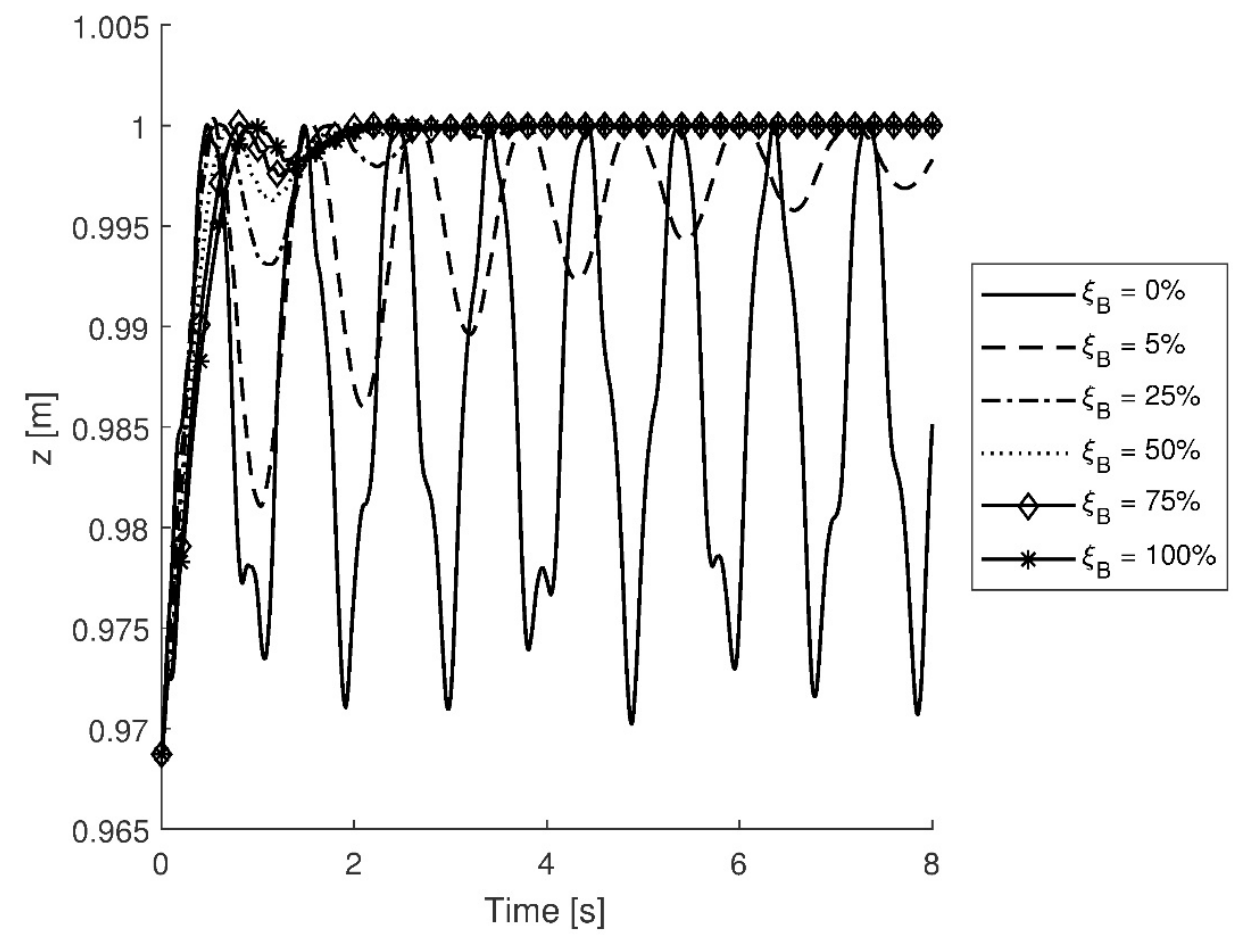

Figure 10: $Z$ position of the free end along the time for different damping ratios

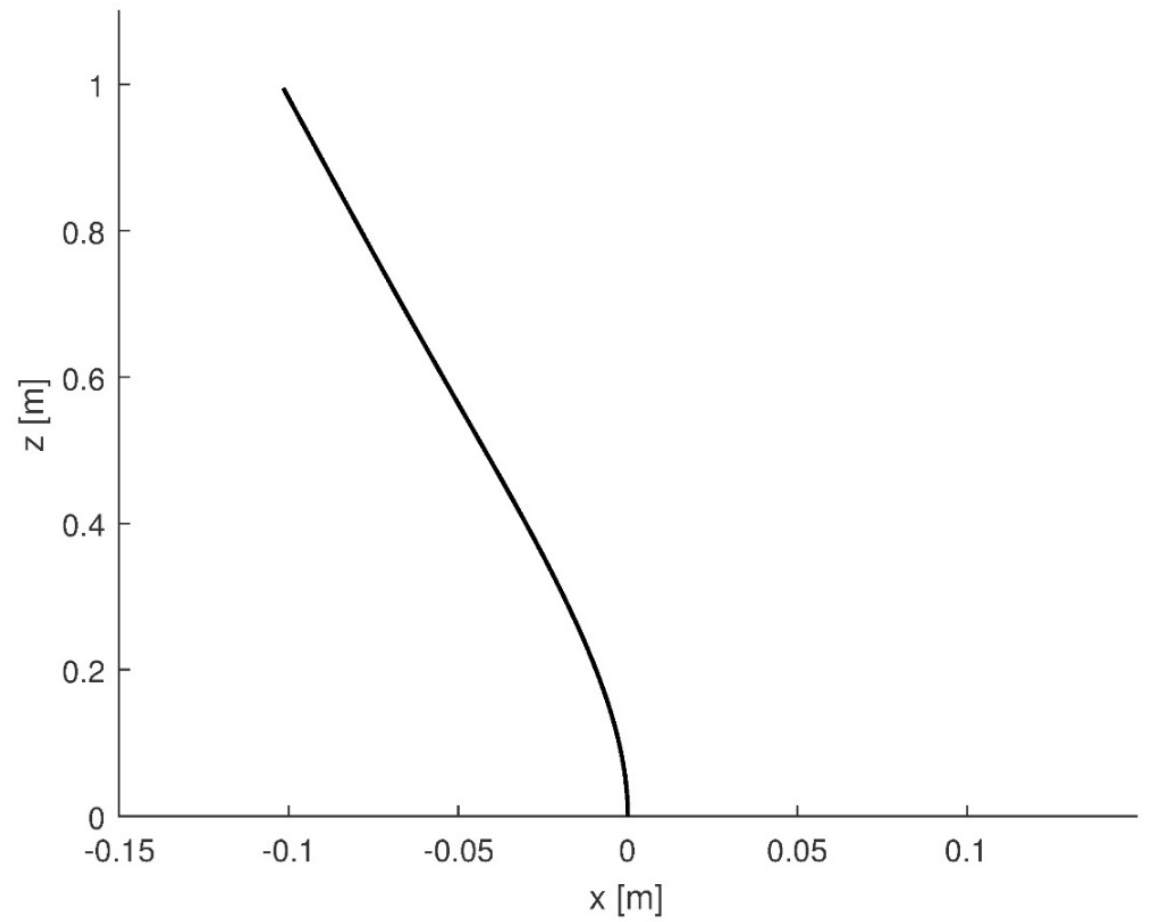

Figure 11:Deformed shape of the column in an instant of the motion 


\section{Experimental validation}

The validation of the model is performed by the simulation of two experimental tests. The first one consists in a free fall cable presented by Koh [33]. The second one consists in a chain catenary mooring line with a fixed end and an imposed motion at the other end presented by Palm et al. [34].

\subsection{Free fall cable}

The free fall cable test consists in a free motion of a cable hanged in one end, as a hinge, and allowing the cable to spin like a pendulum.

The rod used in the experiment was a Neoprene rubber with the properties shown in Table 5. The initial position of the free end of the cable is at the same height of the other end with a horizontal span of 1.8 $\mathrm{m}$. Due to the length of the rod is $2.022 \mathrm{~m}$, the cable bent in its initial position, as can be shown in Figure 12 , a picture of the experimental setup. A damping ratio of $6.2 \%$ was obtained experimentally by a pullback test [33]. Then, the bending critical damping is obtained supposing a double pinned cable element, which leads to $\beta_{B}=\pi / L[36]$.

The simulation was performed with 20 elements for the bar discretization, the time step used in the simulation is $1 \cdot 10^{-3} s$ and the convergence of the dynamics of the cable was achieved after an average of three iterations.

\begin{tabular}{|l|l|}
\hline Parameter & Value \\
\hline Length $[\mathrm{m}]$ & 2.022 \\
\hline Diameter $[\mathrm{m}]$ & 0.03 \\
\hline$A$ & $4.91 \mathrm{E}-04$ \\
\hline $\mathrm{I}$ & $1.92 \mathrm{E}-08$ \\
\hline$\rho\left[\mathrm{kg} / \mathrm{m}^{3}\right]$ & 1430 \\
\hline $\mathrm{E}\left[\mathrm{N} / \mathrm{m}^{2}\right]$ & $3.14 \mathrm{E}+06$ \\
\hline Mass per meter length $[\mathrm{kg} / \mathrm{m}]$ & 0.702 \\
\hline Total mass $[\mathrm{kg}]$ & 1.419 \\
\hline $\mathrm{EA}[\mathrm{N}]$ & $1.541 \mathrm{E}+03$ \\
\hline El $\left[\mathrm{N} \cdot \mathrm{m}^{2}\right]$ & 0.06 \\
\hline$\eta_{B, \text { Critic }}\left[\mathrm{N} /\left(\mathrm{m}^{2} \mathrm{~s}\right)\right]$ & $8.898 \mathrm{E}+06$ \\
\hline$\xi_{B}($ damping ratio $)[\%]$ & 6.2 \\
\hline
\end{tabular}

Table 5: Neoprene rubber cable properties[33] 


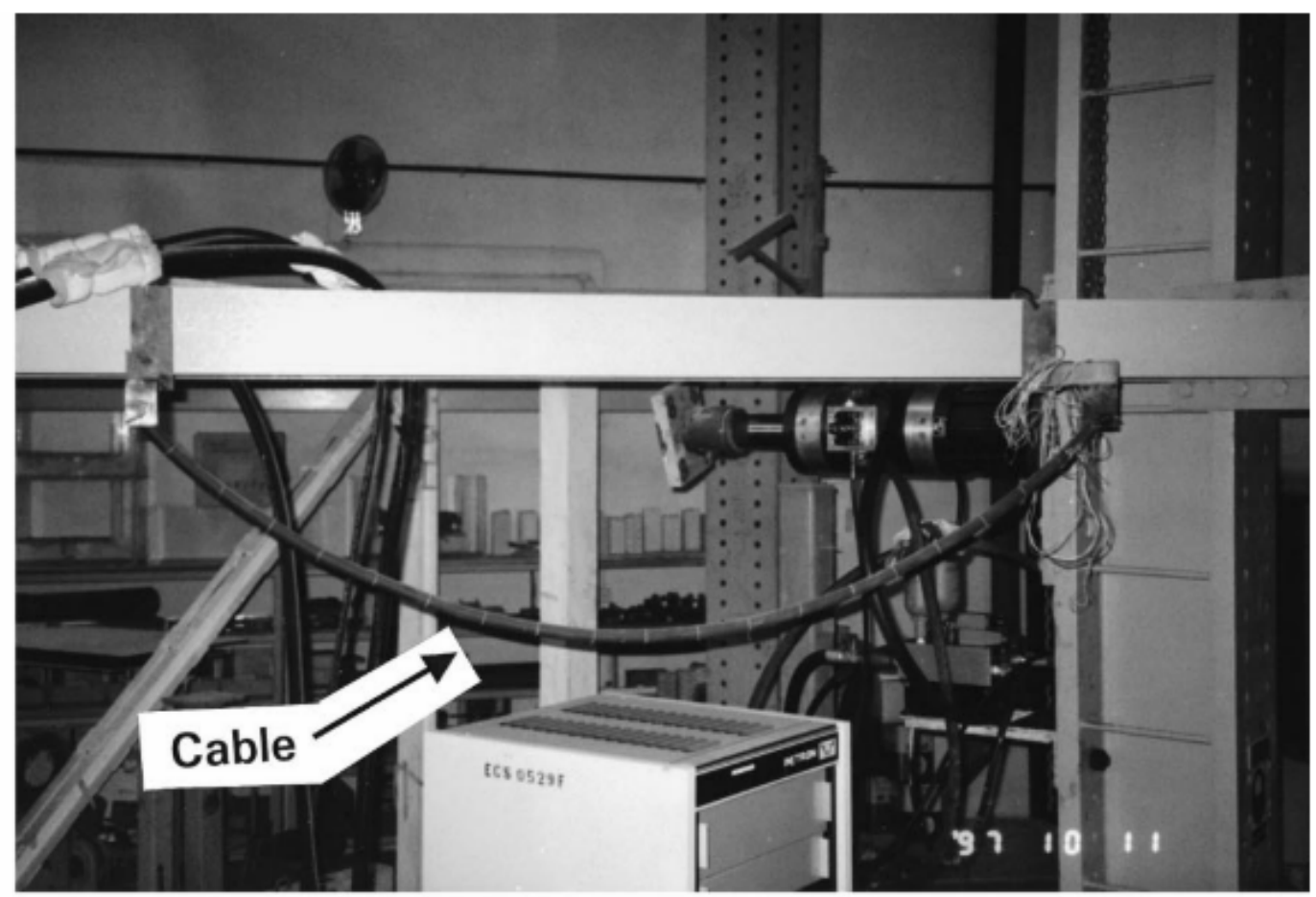

Figure 12: Experimental setup with the cable in its initial position [33]

The experimental results presented by Koh [33] include the dynamic tension of the fixed end obtained by four strain gauges placed symmetrically attached at the surface of the cable near the end, but far enough to prevent the tension concentration due to the fixed support.

Figure 13 and 14 show the comparison of the tension between the experimental results of [33] and the numerical ones obtained with the proposed model. Numerical results fit very well the experimental data. However, the proposed model presents a larger main frequency and gets less accuracy as the time increases (Figure 13 and 14), showing larger amplitudes than the experiment. Then, this could be explained by an additional damping coming from the friction of the hinge at the fixed end and the wires of the strain gages (Figure 12). 


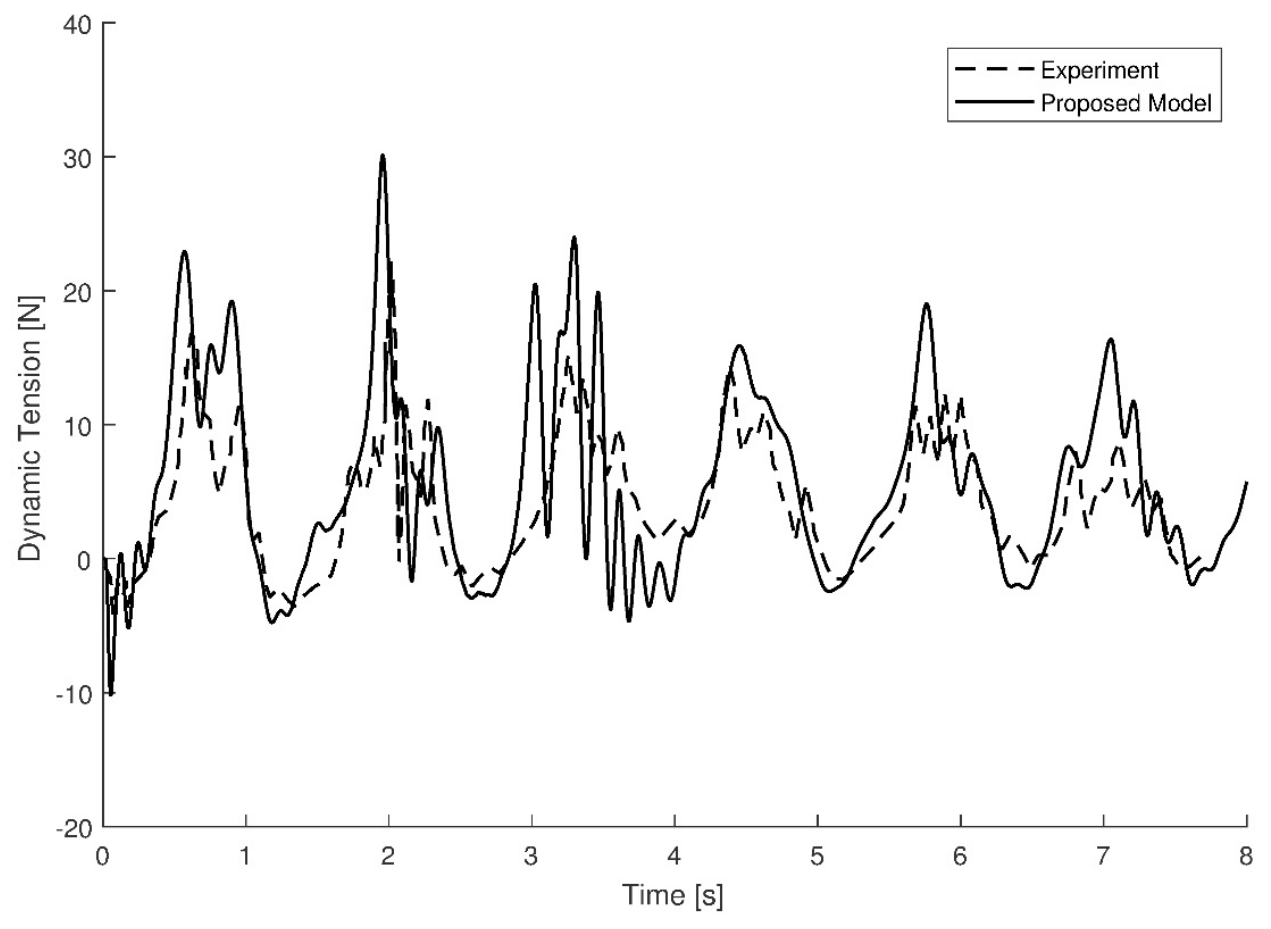

Figure 13: Comparison of experimental and numerical results

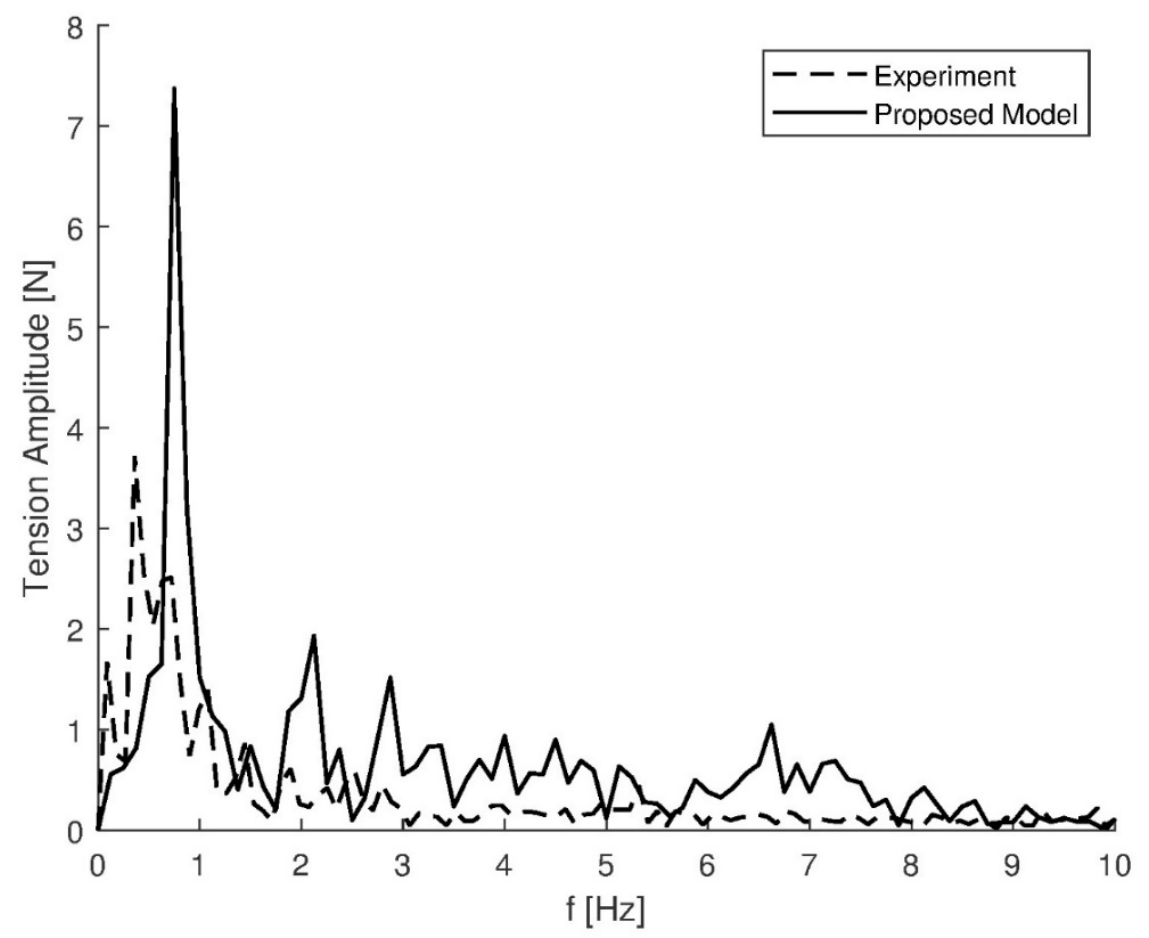

Figure 14: FFT comparison of experimental and numerical results 


\subsection{Mooring chain}

The mooring chain validation consists in a catenary mooring chain fixed on the seabed and attached to a disk at the other end, which spins through a motor equipped with a load cell to get the mooring tension. This model test used was presented by Lindahl [11] and recently represented by Palm et al. [34].

To take into account the hydrodynamic forces, the external force per unit length $\mathbf{q}$ is de fined as:

$$
\mathbf{q}=\mathbf{f}_{h g}+\mathbf{f}_{a m}+\mathbf{f}_{D, t}+\mathbf{f}_{D, n}+\mathbf{f}_{s b}
$$

Where $\mathbf{f}_{h g}$ is the hydrostatic and gravity forces, $\mathbf{f}_{a m}$ is the hydrodynamic inertia force, $\mathbf{f}_{D, t}$ and $\mathbf{f}_{D, n}$ are the hydrodynamic tangential and normal drag forces respectively, and $\mathbf{f}_{s b}$ is the seabed force. These forces per unit length can be expressed as:

$$
\begin{aligned}
& \mathbf{f}_{h g}=\rho_{m} A \mathbf{g}-\rho_{w} A \mathbf{g}=\gamma_{w} \mathbf{e}_{z} \\
& \mathbf{f}_{a m}=-A \rho_{w} C_{a} \ddot{\mathbf{r}}^{n}+A \rho_{w} C_{m} \dot{\mathbf{V}}^{n} \\
& \mathbf{f}_{D, t}=\frac{1}{2} \rho_{w} C_{D, t} d\left|\mathbf{V}^{t}-\dot{\mathbf{r}}^{t}\right|\left(\mathbf{V}^{t}-\dot{\mathbf{r}}^{t}\right) \\
& \mathbf{f}_{D, n}=\frac{1}{2} \rho_{w} C_{D, n} d\left|\mathbf{V}^{n}-\dot{\mathbf{r}}^{n}\right|\left(\mathbf{V}^{n}-\dot{\mathbf{r}}^{n}\right) \\
& \mathbf{f}_{s b, z}=-G_{k} d\left(\mathbf{r}_{z}+\operatorname{depth}\right)-2 G_{c} \sqrt{\rho_{m} A G_{k} d} \min \left(\dot{\mathbf{r}}_{z} ; 0\right) \quad \text { if } \mathbf{r}_{z}+\operatorname{depth}<0 \\
& \mathbf{f}_{s b, y}=-\rho_{m}|\mathbf{g}| \mathrm{G}_{\mu} \min \left(\frac{\dot{\mathbf{r}}_{x y}}{G_{v c}} ; 1\right)
\end{aligned}
$$

The hydrodynamic forces $\mathbf{f}_{a m}, \mathbf{f}_{D, n}, \mathbf{f}_{D, t}$ are based on the Morison's equation for oscillating cylinders [39]. The seabed effect is set as a normal force on the flat sea-bed based on a spring-damper system. The damping force only appears when the node with seabed contact penetrates to the soil (negative velocity), nor when it is coming out. In the Eq. (85) the superscript $n$ and $t$ refer to the normal or the tangent component of the line respectively; $\mathbf{g}$ is the gravity vector, the $\gamma_{w}$ is the wet weight per meter length; the $\mathbf{V}$ is the water particle velocity; the $\dot{\mathbf{V}}$ is the water particle acceleration; the $G_{K}$ is the ballast seabed coefficient or the seabed stiffness in $\left[\mathrm{N} / \mathrm{m}^{3}\right]$; the depth is the sea depth; the $G_{C}$ is the fraction of critical damping of ground, $G_{\mu}$ is the friction coefficient and $G_{v c}$ is the cut-off velocity of friction. The hydrodynamic coefficients are: $C_{D}$ the drag coefficient, $C_{a}$ the added mass coefficient and $C_{m}$ the inertia coefficient.

The properties of the chain and main parameters of the experiment are presented in Table 6 and a sketch of the model test is shown in Figure 15. Moreover, an axial damping ratio of $3 \%$ is set to take into account the friction between links. This value has been set to avoid the use of an artificial numerical damping. The ballast seabed coefficient does not match the value provided by Palm et al. [34], and is set to $3 \mathrm{E}+05 \mathrm{~Pa}$ as set Chen and Basu [22].

The simulation was performed with 30 elements for the chain discretization. The time step used in the simulation are $0.01 \mathrm{~s}$ and $0.005 \mathrm{~s}$ for the $\mathrm{T}=3.5 \mathrm{~s}$ and $\mathrm{T}=1.25 \mathrm{~s}$ disk period, respectively, and the convergence of the dynamics of the chain was achieved after an average of 3 iterations 


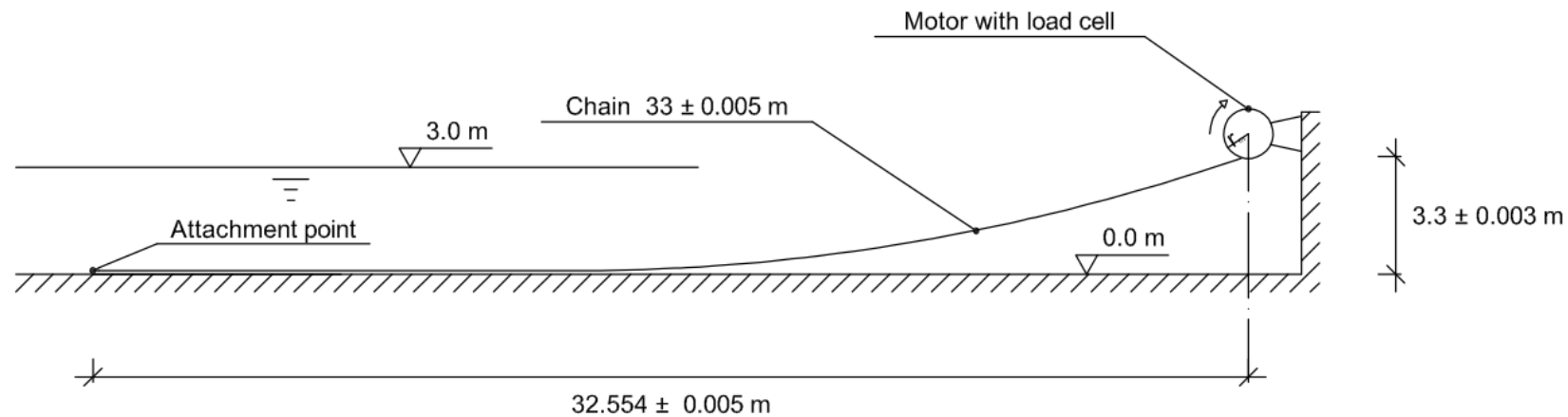

Figure 15: Model test sketch, [34]

\begin{tabular}{|l|l|l|l|}
\hline Line Parameter & Value & Line Parameter & Value \\
\hline Length $[\mathrm{m}]$ & 33 & $\mathrm{Cd}_{\mathrm{n}}$ & 2.5 \\
\hline Diameter $[\mathrm{m}]$ & 0.002 & $\mathrm{Cd}$ & 0.5 \\
\hline Mass per meter length $[\mathrm{kg} / \mathrm{m}]$ & 0.0818 & $\mathrm{Cm}$ & 3.8 \\
\hline Total mass $[\mathrm{kg}]$ & 2.7 & $\rho_{\text {water }}\left[\mathrm{kg} / \mathrm{m}^{3}\right]$ & 1000 \\
\hline Wet weight per meter length $[\mathrm{N} / \mathrm{m}]$ & 0.699 & $G_{k}[\mathrm{~Pa}]$ & $3 \mathrm{E}+05$ \\
\hline EA $[\mathrm{N}]$ & $1.0 \mathrm{E}+04$ & $G_{C}$ & 1 \\
\hline El $\left[\mathrm{N} \cdot \mathrm{m}^{2}\right]$ & 0 & $G_{\mu}$ & 0.3 \\
\hline$\xi_{A}($ damping ratio) $[\%]$ & 3 & $G_{v c}[\mathrm{~m} / \mathrm{s}]$ & 0.01 \\
\hline
\end{tabular}

Table 6: Mooring line properties[34]

The test is performed with two different periods of the movement of the disk: 3.5 and $1.25 \mathrm{~s}$. Figure 16 shows the comparison between the simulations and the experimental results. Overall, the tension at the fairlead of the simulation fits the experimental data in both cases. In addition, the model also captures de loss of tension due to slack events. In the slack events, numerical instabilities can appear due to the loss of the continuity of tension between nodes, which produces snap loads. However, with the proposed model there was no need of numerical damping to prevent these instabilities because the material damping was sufficient to avoid such instabilities. To appraise the latter, Figure 17 shows a cycle tension comparing the behaviour of a chain with $0 \%$ and $3 \%$ of critical damping for the disk period of $1.25 \mathrm{~s}$. 

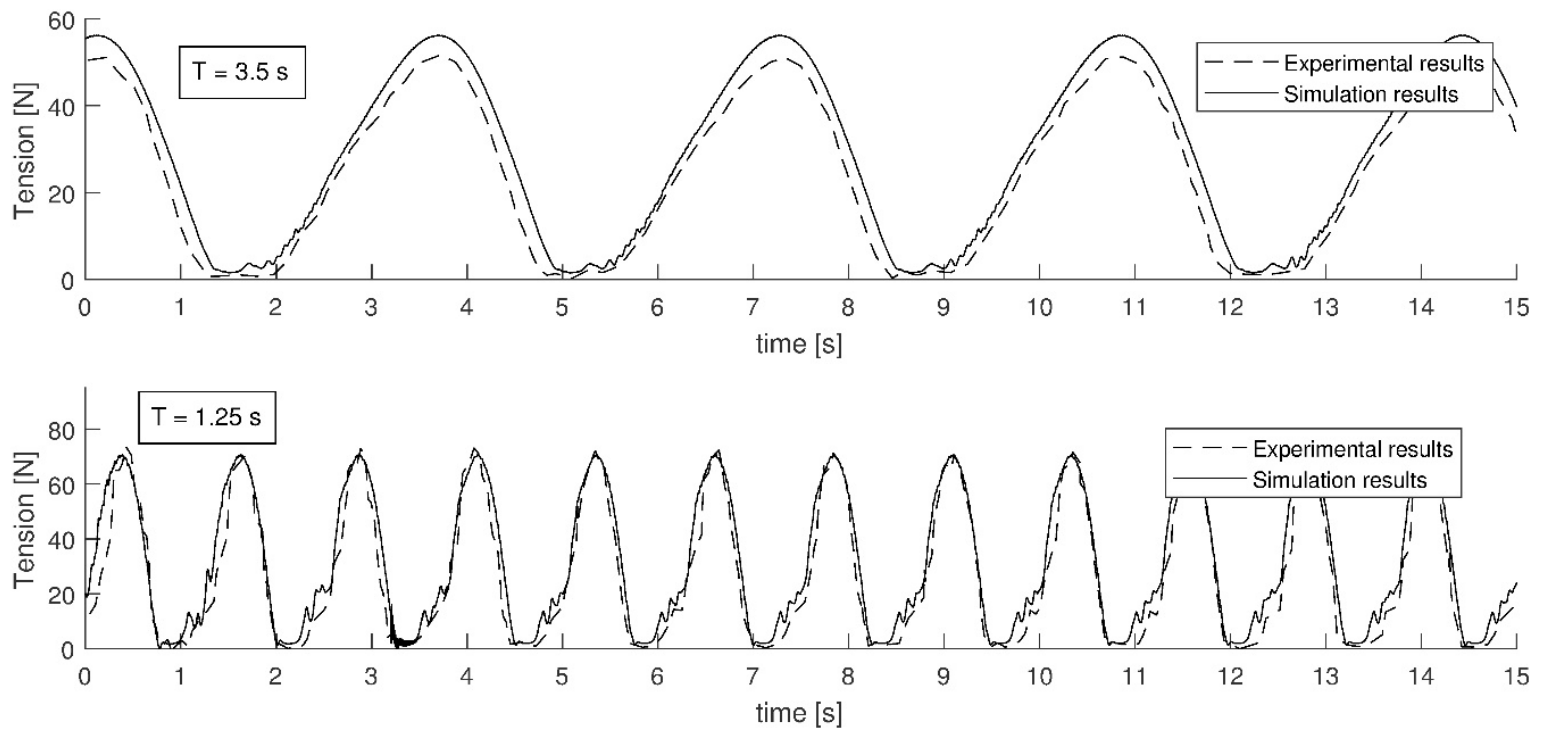

Figure 16: Experimental and simulation results for $T=3.5 \mathrm{~s}$ (above) and $T=1.25 \mathrm{~s}$ (below)

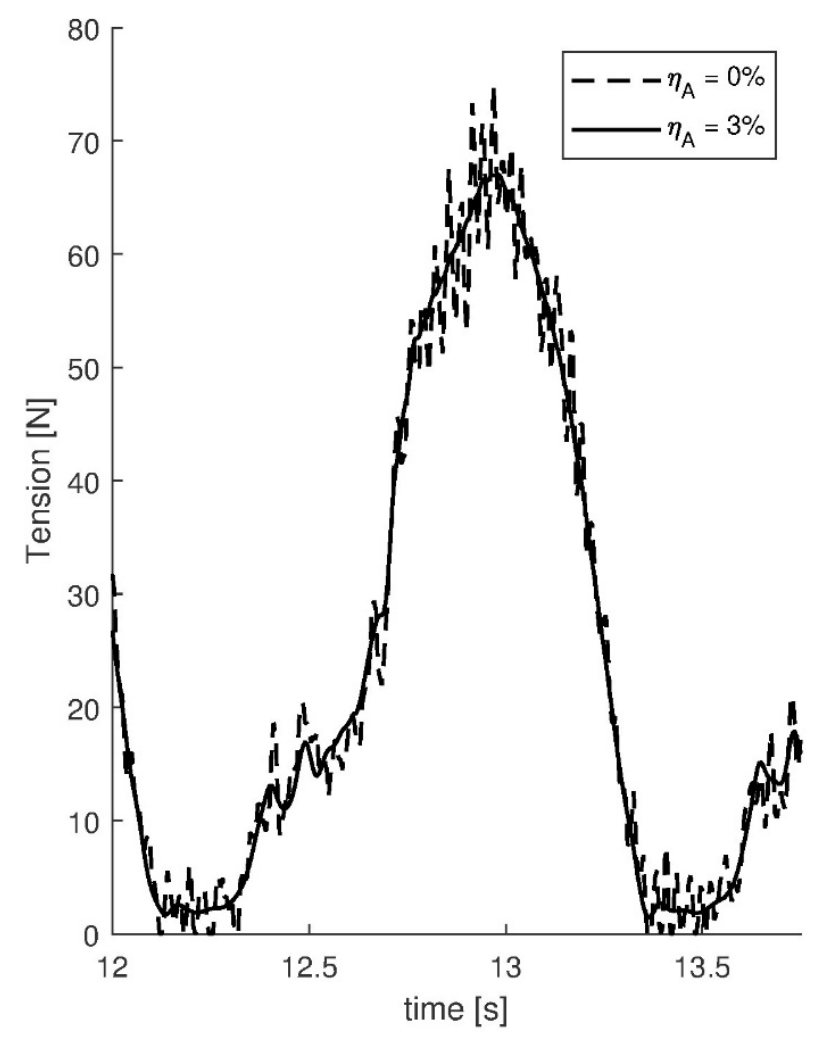

Figure 17: Tension cycle comparison for different values of damping ratio 


\section{Conclusions}

An extension of the Garret rod model [14] is presented including both the line extensibility and the axial and the bending rheological damping. Moreover, the model accounts for the bending and damping terms by the Lagrange multiplier approach. Extending the formulation of the Lagrange multiplier allows distinguishing between the bending and the extensional term in the constitutive equation and relating it with the equation of motion. In this way, the contributions of axial and bending damping are properly assessed and included in the formulation.

The damping of the rods, both the axial and the bending term, are expressed using the Kelvin-Voigt rheological model. This model describes the viscoelasticity with a spring and a dashpot in parallel. The implementation of the rheological damping is introduced in both the equation of motion and the constitutive equation. Also the finite element implementation and the time integration schemes of the new model are presented.

The proposed viscous model efficiently defines the behaviour of visco-elastic materials but also can be used as an energy dissipation mechanism for other internal mechanical interactions of the dynamic system. In this case, the internal friction damping source of stranded cables has to be treated as an equivalent frequency damping.

The model is verified by means of three different tests: an axial free vibration through an initial extension of a cable, a bending free vibration through an initial pure bending state, and a post-buckling behaviour of a column. The simulations produce results that coincide with the analytical ones, despite these examples are highly non-linear, appraising the capacity and the robustness of the model.

The validation of the model is based on the free fall experiment of a cable presented by Koh [33] and the experiment of a mooring chain with an imposed movement in one end presented by Lindahl [11], with no tension episodes. The comparisons show a good agreement between the simulations and the existing experimental results, with a limited amount of iterations. 


\section{References:}

[1] OECD/IEA, "Renewables 2017 Executive Summary," 2017.

[2] WindEurope, "Floating offshore wind energy, a policy blueprint for europe," 2018.

[3] R. James and M. Costa, "Floating Offshore Wind: Market and Technology Review," United Kingdom, 2015.

[4] D. Matha, M. Schlipf, A. Cordle, R. Pereira, and J. Jonkman, "Challenges in Simulation of Aerodynamics, Hydrodynamics , and Mooring-Line Dynamics of Floating Offshore Wind Turbines," Eng. Conf., vol. 8, no. October, pp. 421-428, 2011.

[5] T. S. Walton and H. Polachek, "Calculation of nonlinear transient motion of cables," 1959.

[6] T. Nakajima, S. Motora, and FujinoM., "On the dynamic analysis of multicomponent mooring lines," OTC Pap. 4309, 1982.

[7] C. M. Ablow and S. Schechter, "Numerical simulation of undersea cable dynamics," Ocean Eng., vol. 10, no. 6, pp. 443-457, 1983.

[8] J. J. Burges, "Modeling of undersea cable installation with a finite difference method," in First International of Offshore and Polar Engng Conference, 1991, pp. 2:222-227.

[9] C. T. Howell, "Investigation of the Dynamics of Low-Tension Cables," Massachsetts Institute of Technology, 1992.

[10] J. I. Gobat and M. a. Grosenbaugh, "Application of the generalized- $\alpha$ method to the time integration of the cable dynamics equations," Comput. Methods Appl. Mech. Eng., vol. 190, no. 37-38, pp. 4817-4829, Jun. 2001.

[11] J. Lindahl, “Modellförsök med en förankringskabel. Report Series A:12,” 1985.

[12] O. M. Aamo and T. I. Fossen, "Finite element modelling of mooring lines," Math. Comput. Simul., vol. 53, no. July, pp. 415-422, 2000.

[13] J. Azcona, "Computational and experimental modelling of mooring line dynamics for offshore floating wind turbines," vol. 2016, 2016.

[14] D. L. Garrett, "Dynamic Analysis of Slender Rods," J. Energy Resour. Technol., vol. 104, no. 4, pp. 302-306, 1982.

[15] B. J. Buckham, "Dynamics modelling of low-tension tethers for submerged remotely operated vehicles," University of Victoria, 2003.

[16] Y.-B. Kim, "Dynamic Analysis of multiple-body floating platforms coupled with mooring lines and risers," Texas A\&M University, 2003.

[17] J. Palm, C. Eskilsson, and L. Bergdahl, "An hp-adaptive discontinuous Galerkin method for modelling snap loads in mooring cables," Ocean Eng., vol. 144, no. August, pp. 266-276, 2017.

[18] C. Petrone, N. D. Oliveto, and M. V. Sivaselvan, "Dynamic Analysis of Mooring Cables with Application to Floating Offshore Wind Turbines," J. Eng. Mech., vol. 142, no. 3, p. 04015101, 2016.

[19] H. M. Hilber, T. J. R. Hughes, and R. L. Taylor, "Improved numerical dissipation for time integration 
algorithms in structural dynamics," Earthq. Eng. Struct. Dyn., no. 5, pp. 283-292, 1977.

[20] J. Chung and G. M. Hulbert, "A Time Integration Algorithm for Structural Dynamics With Improved Numerical Dissipation: The Generalized- $\alpha$ Method," J. Appl. Mech., 1993.

[21] J. I. Gobat and M. A. Grosenbaugh, "Time-domain numerical simulation of ocean cable structures," Ocean Eng., vol. 33, no. 10, pp. 1373-1400, 2006.

[22] L. Chen and B. Basu, "Development of an open-source simulation tool for mooring systems Development of an open-source simulation tool for mooring systems," in Ceri-Itrn2018, 2018, no. August.

[23] R. Claren and G. Diana, "Mathematical Analysis of Transmission Line Vibration," IEEE Trans. Power Appar. Syst., vol. PAS-88, no. 12, pp. 1741-1771, Dec. 1969.

[24] C. Hardy, "Analysis of self-damping characteristics of stranded cables in transverse vibrations," in Proceedings of the CSME Mechanical Engineering Forum, 2015, no. January 1990, pp. 117-122.

[25] S. Goudreau, F. Charette, C. Hardy, and L. Cloutier, "Bending Energy Dissipation of Simplified SingleLayer Stranded Cable," J. Eng. Mech., vol. 124, no. 8, pp. 811-817, 2002.

[26] F. Foti, L. Martinelli, and F. Perotti, "A new approach to the definition of self-damping for stranded cables," Meccanica, vol. 51, no. 11, pp. 2827-2845, Nov. 2016.

[27] M. Di Paola, R. Heuer, and A. Pirrotta, "Fractional visco-elastic Euler-Bernoulli beam," Int. J. Solids Struct., vol. 50, no. 22-23, pp. 3505-3510, 2013.

[28] N. D. Oliveto and M. V. Sivaselvan, "3D Finite-Deformation Beam Model with Viscous Damping: Computational Aspects and Applications," J. Eng. Mech., vol. 141, no. 1, p. 04014103, 2015.

[29] O. Martin, "Nonlinear dynamic analysis of viscoelastic beams using a fractional rheological model," Appl. Math. Model., vol. 43, pp. 351-359, 2017.

[30] R. M. Christensen, "Chapter I - Viscoelastic Stress Strain Constitutive Relations," in Theory of Viscoelasticity (Second Edition), Second Edi., R. M. Christensen, Ed. Academic Press, 1982, pp. 134.

[31] J. F. Flory, S. P. Banfield, and D. J. Petruska, "Defining, Measuring, and Calculating the Properties of Fiber Rope Deepwater Mooring Lines," in Offshore Technology Conference, 2004.

[32] S. S. Rao, Mechanical vibrations, vol. 169, no. 4303. Prentice Hall, 2011.

[33] C. G. Koh, Y. Zhang, and S. T. Quek, "Low-Tension Cable Dynamics: Numerical and Experimental Studies," J. Eng. Mech., vol. 125(3), no. March, pp. 347-354, 1999.

[34] J. Palm, G. M. Paredes, F. T. Pinto, and L. Bergdahl, "Simulation of Mooring Cable Dynamics Using a Discontinuous Galerkin Method," in International Conference on Computational Methods in Marine Engineering - MARINE, 2013, pp. 1-12.

[35] T. G. Mezger, The Rheology Handbook, vol. 38, no. 5. 2014.

[36] D. J. Inman, Engineering vibration. 2001.

[37] M. A. Crisfield, Non-linear Finite Element Analysis of Solids and Structures. Volume 2 Advanced Topics. 2000. 
[38] S. P. S. P. Timoshenko and J. J. M. Gere, Theory of elastic stability. 1963.

[39] E. J. Laya, J. J. Connor, and S. S. Sunder, "Hydrodynamic Forces on Flexible Offshore Structures," J. Eng. Mech., vol. 110, no. 3, pp. 433-448, Mar. 1984. 